TRANSACTIONS OF THE

AMERICAN MATHEMATICAL SOCIETY

Volume 361, Number 11, November 2009, Pages 5885-5920

S 0002-9947(09)04779-5

Article electronically published on June 17, 2009

\title{
SURFACE BRANCHED COVERS AND GEOMETRIC 2-ORBIFOLDS
}

\author{
MARIA ANTONIETTA PASCALI AND CARLO PETRONIO
}

\begin{abstract}
Let $\widetilde{\Sigma}$ and $\Sigma$ be closed, connected, and orientable surfaces, and let $f: \widetilde{\Sigma} \rightarrow \Sigma$ be a branched cover. For each branching point $x \in \Sigma$ the set of local degrees of $f$ at $f^{-1}(x)$ is a partition of the total degree $d$. The total length of the various partitions is determined by $\chi(\widetilde{\Sigma}), \chi(\Sigma), d$ and the number of branching points via the Riemann-Hurwitz formula. A very old problem asks whether a collection of partitions of $d$ having the appropriate total length (that we call a candidate cover) always comes from some branched cover. The answer is known to be in the affirmative whenever $\Sigma$ is not the 2 -sphere $S$, while for $\Sigma=S$ exceptions do occur. A long-standing conjecture however asserts that when the degree $d$ is a prime number a candidate cover is always realizable. In this paper we analyze the question from the point of view of the geometry of 2-orbifolds, and we provide strong supporting evidence for the conjecture. In particular, we exhibit three different sequences of candidate covers, indexed by their degree, such that for each sequence:

- The degrees giving realizable covers have asymptotically zero density in the naturals.

- Each prime degree gives a realizable cover.
\end{abstract}

\section{INTRODUCTION}

This paper is devoted to a geometric approach, based on 2-orbifolds, to the Hurwitz existence problem for branched covers between surfaces; see [10] for the original source, the classical [11, 22, 21, 5, 6, 4, 13, 3, 7, 14, 12, the more recent 1, 15, 16, 18, 19, 17, 28, and below. We determine the realizability of all candidate surface branched covers having associated candidate covers between 2-orbifolds with non-negative Euler characteristic. This yields a complete analysis of the existence of several infinite series of candidate covers and in particular to theorems giving rather surprising connections with number-theoretic facts. These results provide in particular strong support for the long-standing conjecture [3] that a candidate cover with prime total degree is always realizable. The appropriate terminology and the statements of some of the results established below are given in the rest of the present Introduction. Our main results (Theorems 0.4 to 0.6 ) will not involve explicit reference to 2-orbifolds.

Branched covers. Let $\widetilde{\Sigma}$ and $\Sigma$ be closed, connected, and orientable surfaces, and $f: \widetilde{\Sigma} \rightarrow \Sigma$ be a branched cover, i.e. a map locally modelled on functions of the form $(\mathbb{C}, 0) \stackrel{z \mapsto z^{k}}{\longrightarrow}(\mathbb{C}, 0)$ with $k \geqslant 1$. If $k>1$, then 0 in the target $\mathbb{C}$ is a branching

Received by the editors September 17, 2007.

2000 Mathematics Subject Classification. Primary 57M12; Secondary 57M50.

(C)2009 American Mathematical Society Reverts to public domain 28 years from publication 
point, and $k$ is the local degree at 0 in the source $\mathbb{C}$. There is a finite number $n$ of branching points, and, removing all of them from $\Sigma$ and their preimages from $\widetilde{\Sigma}$, we see that $f$ induces a genuine cover of some degree $d$. The collection $\left(d_{i j}\right)_{j=1}^{m_{i}}$ of the local degrees at the preimages of the $i$-th branching point is a partition $\Pi_{i}$ of $d$. We now define:

- $\ell\left(\Pi_{i}\right)$ to be the length $m_{i}$ of $\Pi_{i}$;

- $\Pi$ as the set $\left\{\Pi_{1}, \ldots, \Pi_{n}\right\}$ of all partitions of $d$ associated to $f$;

- $\ell(\Pi)$ to be the total length $\ell\left(\Pi_{1}\right)+\ldots+\ell\left(\Pi_{n}\right)$ of $\Pi$.

Then the multiplicativity of the Euler characteristic $\chi$ under genuine covers for surfaces with boundary implies the classical Riemann-Hurwitz formula

$$
\chi(\widetilde{\Sigma})-\ell(\Pi)=d \cdot(\chi(\Sigma)-n) .
$$

Candidate branched covers and the realizability problem. Consider again two closed, connected, and orientable surfaces $\widetilde{\Sigma}$ and $\Sigma$, integers $d \geqslant 2$ and $n \geqslant 1$, and a set of partitions $\Pi=\left\{\Pi_{1}, \ldots, \Pi_{n}\right\}$ of $d$, with $\Pi_{i}=\left(d_{i j}\right)_{j=1}^{m_{i}}$, such that condition (11) is satisfied. We associate to these data the symbol

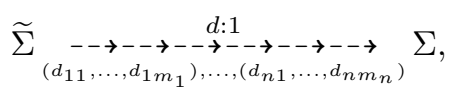

that we will call a candidate surface branched cover. A classical (and still not completely solved) problem, known as the Hurwitz existence problem, asks which candidate surface branched covers are actually realizable, namely induced by some existent branched cover $f: \widetilde{\Sigma} \rightarrow \Sigma$. A non-realizable candidate surface branched cover will be called exceptional.

Remark 0.1 . The symbol $\left(\widetilde{\Sigma}, \Sigma, n, d,\left(d_{i j}\right)\right)$ and the name compatible branch datum are used in [18, 19] instead of the terminology of "candidate covers" we will use here.

Known results. Over the last 50 years the Hurwitz existence problem was the object of many papers, already listed above. The combined efforts of several mathematicians led in particular to the following results [11, 3]:

- If $\chi(\Sigma) \leqslant 0$, then any candidate surface branched cover is realizable; i.e., the Hurwitz existence problem has a positive solution in this case.

- If $\chi(\Sigma)>0$, i.e., if $\Sigma$ is the 2 -sphere $S$, there exist exceptional candidate surface branched covers.

Remark 0.2. A version of the Hurwitz existence problem exists also for possibly non-orientable $\widetilde{\Sigma}$ and $\Sigma$. Condition (1) must be complemented in this case with a few more requirements (some obvious, and one slightly less obvious, see [18]). However it has been shown [4, 3] that again this generalized problem always has a positive solution if $\chi(\Sigma) \leqslant 0$, and that the case where $\Sigma$ is the projective plane reduces to the case where $\Sigma$ is the 2-sphere $S$.

According to these facts, in order to face the Hurwitz existence problem, it is not restrictive to assume the candidate covered surface $\Sigma$ is the 2-sphere $S$, which we will do henceforth. Considerable energy has been devoted over time to a general understanding of the exceptional candidate surface branched covers in this case, and quite a lot of progress has been made (see for instance the survey of known results contained in [18, together with the later papers [19, 17, 28]), but the global pattern 
remains elusive. In particular the following conjecture proposed in [3] appears to still be open:

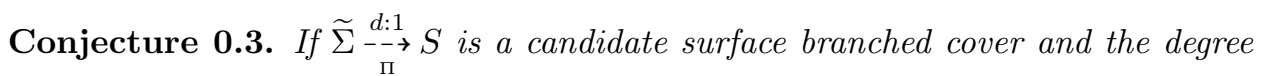
$d$ is a prime number, then the candidate is realizable.

We mention in particular that all exceptional candidate surface branched covers with $n=3$ and $d \leqslant 20$ have been determined by computer in [28. There are very many of them, but none occurs for prime $d$.

Some instances of the Hurwitz existence problem are treated in [25, 26, 27], where a connection is also shown with the Mason-Stother "abc-theorem" for polynomials. The methods used in these papers are combinatorial constructions and the Riemann Existence Theorem. We also address the reader to [9]. More relations of our results with those of these papers are discussed in [2].

Main new results. Using the geometry of Euclidean 2-orbifolds we will establish in this paper (among others) the next three theorems. Recall that a candidate

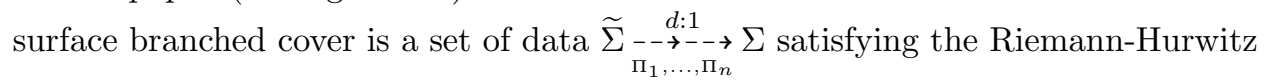
condition (11). Moreover $S$ denotes the 2-sphere.

Theorem 0.4. Suppose $d=4 k+1$ for $k \in \mathbb{N}$. Then

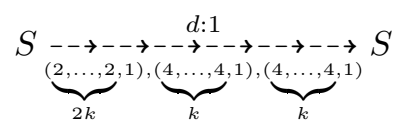

is a candidate surface branched cover, and it is realizable if and only if $d$ can be expressed as $x^{2}+y^{2}$ for some $x, y \in \mathbb{N}$.

Theorem 0.5. Suppose $d=6 k+1$ for $k \in \mathbb{N}$. Then

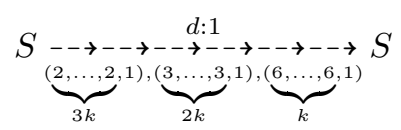

is a candidate surface branched cover and it is realizable if and only if $d$ can be expressed as $x^{2}+x y+y^{2}$ for some $x, y \in \mathbb{N}$.

Theorem 0.6. Suppose $d=3 k+1$ for $k \in \mathbb{N}$. Then

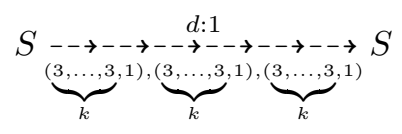

is a candidate surface branched cover and it is realizable if and only if $d$ can be expressed as $x^{2}+x y+y^{2}$ for some $x, y \in \mathbb{N}$.

What makes these results remarkable in view of Conjecture 0.3 is that:

- A prime number of the form $4 k+1$ can always be expressed as $x^{2}+y^{2}$ for $x, y \in \mathbb{N}$ (Fermat).

- A prime number of the form $6 k+1$ (or equivalently $3 k+1$ ) can always be expressed as $x^{2}+x y+y^{2}$ for $x, y \in \mathbb{N}$ (Gauss).

- The integers that can be expressed as $x^{2}+y^{2}$ or as $x^{2}+x y+y^{2}$ with $x, y \in \mathbb{N}$ have asymptotically zero density in $\mathbb{N}$. 
This means that a candidate cover in any of our three statements is "exceptional with probability 1", even though it is realizable when its degree is prime. Note also that it was shown in 3 , that establishing Conjecture 0.3 in the special case of three branching points would imply the general case.

Associated candidate 2-orbifold covers. A 2-orbifold $X=\Sigma\left(p_{1}, \ldots, p_{n}\right)$ is a closed orientable surface $\Sigma$ with $n$ cone points of orders $p_{i} \geqslant 2$, at which $X$ has a singular differentiable structure given by the quotient $\mathbb{C} /\left\langle\operatorname{rot}\left(2 \pi / p_{i}\right)\right\rangle$. Bill Thurston [23] introduced the notions (reviewed below) of orbifold cover and orbifold Euler characteristic

$$
\chi^{\mathrm{orb}}\left(\Sigma\left(p_{1}, \ldots, p_{n}\right)\right)=\chi(\Sigma)-\sum_{i=1}^{n}\left(1-\frac{1}{p_{i}}\right),
$$

designed so that if $f: \widetilde{X} \stackrel{d: 1}{\longrightarrow} X$ is an orbifold cover, then $\chi^{\text {orb }}(\widetilde{X})=d \cdot \chi^{\text {orb }}(X)$. He also showed that:

- If $\chi^{\text {orb }}(X)>0$, then $X$ is either bad (not covered by a surface in the sense of orbifolds) or spherical, namely the quotient of the metric 2-sphere $\mathbb{S}$ under a finite isometric action.

- If $\chi^{\mathrm{orb}}(X)=0$ (respectively, $\chi^{\mathrm{orb}}(X)<0$ ), then $X$ is Euclidean (respectively, hyperbolic), namely the quotient of the Euclidean plane $\mathbb{E}$ (respectively, the hyperbolic plane $\mathbb{H}$ ) under a discrete isometric action.

As pointed out in 18 and spelled out below, any candidate surface branched

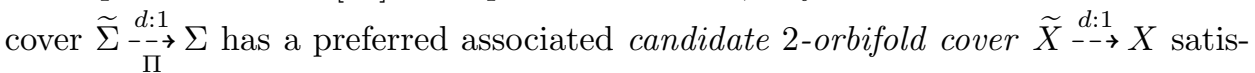

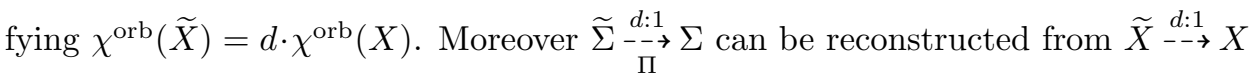
if some additional covering instructions are provided.

More new results. The main idea of this paper is to analyze the realizability of

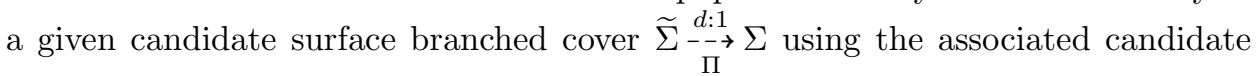
2-orbifold cover $\widetilde{X} \stackrel{d: 1}{\rightarrow \rightarrow} X$ and the geometries of $\widetilde{X}$ and $X$. It turns out that this is particularly effective when $\chi^{\text {orb }}(X)$ is non-negative (note that $\chi^{\text {orb }}(\widetilde{X})$ has the same sign as $\chi^{\text {orb }}(X)$, being $d$ times it), but we will also briefly touch the case $\chi^{\text {orb }}(X)<0$. In the bad $/$ spherical case the statement is quite expressive:

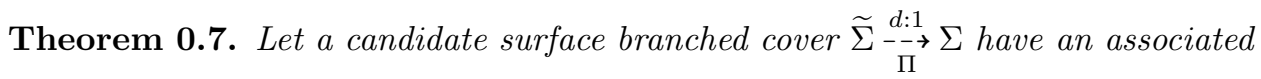

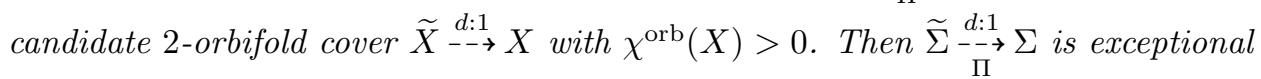
if and only if $\tilde{X}$ is bad and $X$ is spherical. All exceptions occur with non-prime degree.

Turning to the Euclidean case (which leads in particular to Theorems 0.4 to 0.6 ) we confine ourselves here to the following informal:

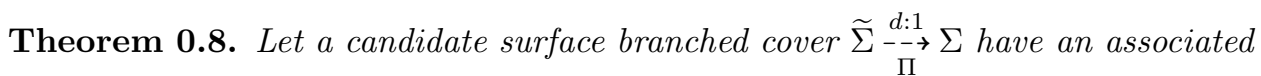
candidate 2 -orbifold cover $\widetilde{X}_{\stackrel{d: 1}{d} \rightarrow X}$ with Euclidean $X$. Then its realizability can be decided explicitly in terms of $d$ and $\Pi$. More precisely, as in Theorems 0.4 to 0.6 , given $\Pi$ the condition on $d$ depends on a congruence and/or an integral quadratic form. No exceptions occur when $d$ is a prime number. 
We conclude with our statement for the hyperbolic case. A 2-orbifold is called triangular if it has the form $S(p, q, r)$.

Theorem 0.9. There exist 9 candidate surface branched covers having an associated candidate 2 -orbifold cover $\widetilde{X} \rightarrow X$ with $\widetilde{X}$ and $X$ being hyperbolic triangular orbifolds. All of them but two are realizable. Exceptions occur in degrees 8 and 16 (which are not prime).

For the case of non-negative $\chi^{\text {orb }}$, within the proofs of Theorems 0.7 and 0.8 we will describe explicit geometric constructions of all the realizable covers. The proof of Theorem 0.9 has instead a more combinatorial flavour.

Remark 0.10. A geometric interpretation of our results is worth pointing out. Let $\widetilde{\Sigma}-\rightarrow \Sigma$ be a candidate surface branched cover with associated candidate orbifold cover $\widetilde{X} \rightarrow X$, and suppose that $\widetilde{X}$ and $X$ are geometric. Then we have identifications $\widetilde{X}=\mathbb{X} / \widetilde{\Gamma}$ and $X=\mathbb{X} / \Gamma$, where $\mathbb{X}$ is one of the model geometries $\mathbb{S}, \mathbb{E}$ or $\mathbb{H}$ (the same for $\widetilde{X}$ and $X$ ), and $\widetilde{\Gamma}, \Gamma$ are discrete cocompact groups of isometries of $\mathbb{X}$. A realization of the cover then corresponds to an identification of $\widetilde{\Gamma}$ to a subgroup of $\Gamma$. Our results in the spherical, Euclidean, and hyperbolic cases therefore yield a classification of the inclusions, respectively, between finite subgroups of $\mathrm{SO}(3)$, between 2-dimensional crystallographic groups, and between discrete, cocompact subgroups of $\operatorname{PSL}(2 ; \mathbb{R})$ with no more than three singular orbits.

\section{A geometric Approach to the PROBlem}

In this section we will describe the general framework leading to the proofs of Theorems 0.4 to 0.8 , carried out in Sections 2 and 3 .

Orbifold covers. Besides the terminology and facts on 2-orbifolds already mentioned in the Introduction (and not reviewed here) we will need the precise definition of a degree- $d$ cover $f: \widetilde{X} \rightarrow X$ between 2-orbifolds. This is a map such that $f^{-1}(x)$ generically consists of $d$ points and locally making a diagram of the following form commutative:

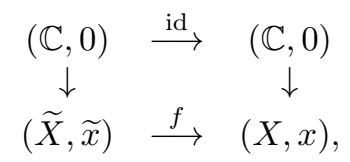

where $\widetilde{x}$ and $x$ have cone orders $\widetilde{p}$ and $p=k \cdot \widetilde{p}$ respectively, and the vertical arrows are the projections corresponding to the actions of $\langle\operatorname{rot}(2 \pi / \widetilde{p})\rangle$ and $\langle\operatorname{rot}(2 \pi / p)\rangle$, namely the maps defining the (possibly singular) local differentiable structures at $\widetilde{x}$ and $x$. Since this local model can be described by the map $z \mapsto z^{k}$, we see that $f$ induces a branched cover between the underlying surfaces of $\widetilde{X}$ and $X$. Using the Riemann-Hurwitz formula (1), it is then easy to show that $\chi^{\operatorname{orb}}(\widetilde{X})=d \cdot \chi^{\operatorname{orb}}(X)$.

Bill Thurston introduced in 23] the notion of orbifold universal cover and established its existence. Using this fact we easily get the following result that we will need below (where, not surprisingly, good means "not bad"):

Lemma 1.1. If $\widetilde{X}$ is bad and $X$ is good, then there cannot exist any orbifold cover $\widetilde{X} \rightarrow X$. 
Preferred associated orbifold cover. As one easily sees, distinct orbifold covers can induce the same surface branched cover (in the local model, the two cone orders can be multiplied by one and the same integer). However a surface branched cover has an "easiest" associated orbifold cover, i.e. that with the smallest possible cone orders. This carries over to candidate covers, as we will now spell out. Consider a candidate surface branched cover

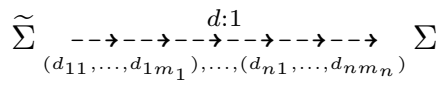

and define

$$
\begin{array}{ll}
p_{i}=\text { l.c.m. }\left\{d_{i j}: j=1, \ldots, m_{i}\right\}, & p_{i j}=p_{i} / d_{i j}, \\
X=\Sigma\left(p_{1}, \ldots, p_{n}\right), & \widetilde{X}=\widetilde{\Sigma}\left(\left(p_{i j}\right)_{i=1, \ldots, n}^{j=1, \ldots, m_{i}}\right),
\end{array}
$$

where "l.c.m." stands for "least common multiple". Then we have a preferred associated candidate 2 -orbifold cover $\widetilde{X} \stackrel{d: 1}{\rightarrow} X$ satisfying $\chi^{\text {orb }}(\widetilde{X})=d \cdot \chi^{\text {orb }}(X)$. Note that the original candidate surface branched cover cannot be reconstructed from $\widetilde{X}, X, d$ alone, but it can if $\widetilde{X} \stackrel{d: 1}{\rightarrow} X$ is complemented with the covering instructions

$$
\left(p_{11}, \ldots, p_{1 m_{1}}\right) \rightarrow p_{1}, \quad \ldots \quad\left(p_{n 1}, \ldots, p_{n m_{n}}\right) \rightarrow p_{n}
$$

that we will sometimes include in the symbol $\tilde{X}_{\stackrel{d: 1}{d} \rightarrow X}$ itself, omitting the $p_{i j}$ 's equal to 1 . Of course a candidate surface branched cover is realizable if and only if the associated candidate 2-orbifold cover with appropriate covering instructions is realizable.

The geometric approach. To analyze the realizability of a candidate surface branched cover we will switch to the associated candidate 2-orbifold cover $\widetilde{X} \rightarrow X$ and we will use geometry either to explicitly construct a map $f: \widetilde{X} \rightarrow X$ realizing it, or to show that such an $f$ cannot exist.

To explain how this works we first note that any 2-orbifold $X$ with a fixed geometric structure of type $\mathbb{X} \in\{\mathbb{S}, \mathbb{E}, \mathbb{H}\}$ has a well-defined distance function. This is because the structure is given by a quotient map $\mathbb{X} \rightarrow X$, that for obvious reasons we will call a geometric universal cover of $X$, defined by an isometric and discrete (even if not free) action. Therefore a piecewise smooth path $\alpha$ in $X$ has a well-defined length obtained by lifting it to a path $\widetilde{\alpha}$ in $\mathbb{X}$, even if $\widetilde{\alpha}$ itself is not unique (even up to automorphisms of $\widetilde{X}$ ) when $\alpha$ goes through some cone point of $X$. Now we have the following:

Proposition 1.2. Let $f: \widetilde{X} \rightarrow X$ be a 2-orbifold cover. Suppose that $X$ has a fixed geometry with geometric universal cover $\pi: \mathbb{X} \rightarrow X$. Then there exists a geometric structure on $\widetilde{X}$ with geometric universal cover $\widetilde{\pi}: \mathbb{X} \rightarrow \widetilde{X}$ and an isometry $\tilde{f}: \mathbb{X} \rightarrow \mathbb{X}$ such that $\pi \circ \tilde{f}=f \circ \tilde{\pi}$.

Proof. We define the length of a path in $\widetilde{X}$ as the length of its image in $X$ under $f$, and we consider the corresponding distance. Analyzing the local model of $f$, one sees that this distance is compatible with a local orbifold geometric structure also of type $\mathbb{X}$, so there is one global such structure on $\widetilde{X}$, with geometric universal cover $\tilde{\pi}: \mathbb{X} \rightarrow \widetilde{X}$. The properties of the universal cover imply that there is a map $\tilde{f}: \mathbb{X} \rightarrow \mathbb{X}$ such that $\pi \circ \widetilde{f}=f \circ \widetilde{\pi}$. By construction $\widetilde{f}$ preserves the length of paths, but $\mathbb{X}$ is a manifold, not an orbifold, so $\widetilde{f}$ is a local isometry. In particular 
it is a cover, but $\mathbb{X}$ is simply connected, so $\tilde{f}$ is a homeomorphism and hence an isometry.

Any spherical 2-orbifold $X$ is rigid, namely the geometric universal cover $\mathbb{S} \rightarrow X$ is unique up to automorphisms of $\mathbb{S}$ and $X$, so in the spherical case one is not faced with any choice while applying Proposition 1.2. On the contrary Euclidean 2 -orbifolds are never rigid, since the metric can always be rescaled (and it can also be changed in more essential ways on the torus $T$ and on $S(2,2,2,2)$; see below). In this case we will slightly modify the content of Proposition 1.2 by rescaling $\widetilde{X}$ so that its area equals that of $X$, in which case $\tilde{f}$ is no more an isometry but merely a complex-affine map $\mathbb{C} \rightarrow \mathbb{C}$, with $\mathbb{C}$ identified to $\mathbb{E}$. More precisely:

Proposition 1.3. Let $f: \widetilde{X} \stackrel{d: 1}{\longrightarrow} X$ be a 2-orbifold cover. Suppose that $X$ has a fixed Euclidean structure with geometric universal cover $\pi: \mathbb{E} \rightarrow X$. Then there exists a Euclidean structure on $\widetilde{X}$ with geometric universal cover $\tilde{\pi}: \mathbb{E} \rightarrow \widetilde{X}$ such that $X$ and $\widetilde{X}$ have the same area, and a map $\widetilde{f}: \mathbb{E} \rightarrow \mathbb{E}$ of the form $\tilde{f}(z)=\lambda \cdot z+\mu$, with $\lambda, \mu \in \mathbb{C}$, such that $\pi \circ \tilde{f}=f \circ \tilde{\pi}$. This implies that $d=|\lambda|^{2}$.

Proof. With respect to the structure on $\widetilde{X}$ given by Proposition 1.2 the area of $\widetilde{X}$ is $d$ times that of $X$, so the scaling factor is $1 / \sqrt{d}$. After rescaling, $\widetilde{f}$ is therefore $\sqrt{d}$ times an isometry, and the conclusion follows.

We remark here that, despite being rather elementary, the previous proposition is the main technical tool underlying our main results, leading to the new restrictions on the realizability of candidate branched covers in the Euclidean case.

To conclude the section, we note that for candidate covers of the form $\underset{\Pi}{S \underset{\Pi}{d: 1} S} S$ with three branching points, as most of ours will be, the Riemann-Hurwitz formula (1) reads

$$
\ell(\Pi)=d+2 \text {. }
$$

\section{Positive Euler Characteristic}

In this section we will establish Theorem 0.7. More precisely we will show:

Theorem 2.1. A candidate surface branched cover $\widetilde{\Sigma} \rightarrow \Sigma$ having an associated candidate 2 -orbifold cover $\widetilde{X} \rightarrow X$ with $\chi^{\text {orb }}(X)>0$ is exceptional if and only if $\widetilde{X}$ $i s$ bad and $X$ is spherical. This occurs precisely for the following candidate covers, in none of which the degree is prime:

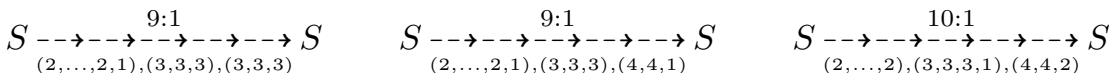

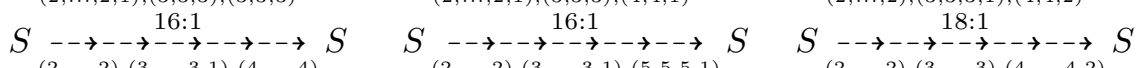

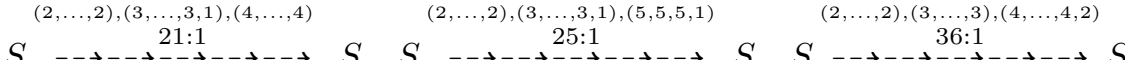

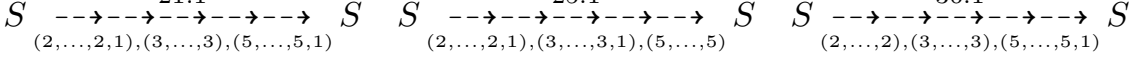

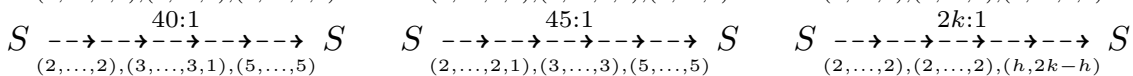

with $k>h \geqslant 1$ in the last item.

In addition to proving this result we will describe all $\widetilde{\Sigma} \rightarrow \Sigma$ having associated $\widetilde{X} \rightarrow X$ with $\chi^{\text {orb }}(X)>0$ not listed in the statement, and we will explicitly construct a geometric realization of each such $\widetilde{X} \rightarrow X$. To outline our argument, we 
first recall that the 2-orbifolds $X$ with $\chi^{\text {orb }}(X)>0$ are

$$
S, \quad S(p), \quad S(p, q), \quad S(2,2, p), \quad S(2,3,3), \quad S(2,3,4), \quad S(2,3,5) .
$$

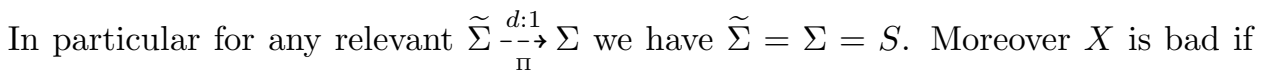
and only if it is $S(p)$ for $p>1$ or $S(p, q)$ for $p \neq q>1$, and in all other cases it has a rigid spherical structure. Our main steps will be as follows:

- We will determine all the candidate surface branched covers having an associated candidate $\widetilde{X} \rightarrow X$ with positive $\chi^{\text {orb }}(\widetilde{X})$ and $\chi^{\text {orb }}(X)$, and the corresponding covering instructions for $\widetilde{X} \rightarrow X$.

- For each spherical $X$ with $\chi^{\text {orb }}(X)>0$ we will explicitly describe (and fix) the geometric universal cover $\pi: \mathbb{S} \rightarrow X$.

- For each $\widetilde{X} \rightarrow X$ with $\chi^{\text {orb }}(X)>0$ (complemented with its covering instructions) associated to some candidate surface branched cover, except when $\widetilde{X}$ is bad and $X$ is spherical, we will explicitly describe an isometry $\widetilde{f}: \mathbb{S} \rightarrow \mathbb{S}$ such that there exists $f: \widetilde{X} \rightarrow X$ realizing $\widetilde{X} \rightarrow X$ with $\pi \circ \tilde{f}=f \circ \widetilde{\pi}$, where $\pi$ and $\widetilde{\pi}$ are the geometric universal covers of $\widetilde{X}$ and $X$ described in the previous step.

Remark 2.2. A description of the realizations of all the relevant candidate covers not listed as exceptional in Theorem 2.1 will be given below in Propositions 2.9-2.11 by explicit geometric constructions. A few figures elucidating these constructions will also be provided; more illustrations (with color) are available on the web [20]. We will also complement these results by exhibiting explicit permutations that yield the same realizations in the original algebraic spirit of [10. We thank the referee for suggesting this addition.

Relevant candidate covers. To list all candidate surface branched covers having an associated candidate $\widetilde{X} \rightarrow X$ with positive $\chi^{\text {orb }}(X)$ our steps will be as follows:

- We consider all possible pairs $(\widetilde{X}, X)$ such that $\chi^{\mathrm{orb}}(\widetilde{X}) / \chi^{\mathrm{orb}}(X)$ is an integer $d>1$.

- Supposing $X$ has $n$ cone points of orders $p_{1}, \ldots, p_{n}$, we consider all possible ways of grouping the orders of the cone points of $\widetilde{X}$ as

$$
\left(q_{11}, \ldots, q_{1 \mu_{1}}\right), \ldots,\left(q_{n 1}, \ldots, q_{n \mu_{n}}\right)
$$

so that $q_{i j}$ divides $p_{i}$ for all $i$ and $j$.

- We determine $m_{i} \geqslant \mu_{i}$ so that, setting $q_{i j}=1$ for $j>\mu_{i}$ and $d_{i j}=\frac{p_{i}}{q_{i j}}$, we have that $\sum_{j=1}^{m_{i}} d_{i j}$ is equal to $d$ for all $i$.

- We check that $p_{i}$ is the least common multiple of $\left(d_{i j}\right)_{j=1}^{m_{i}}$.

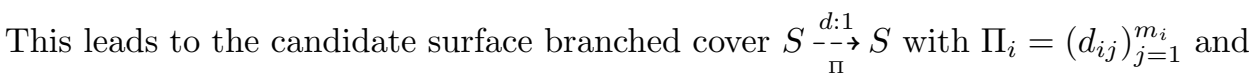
$\Pi=\left(\Pi_{i}\right)_{i=1}^{n}$, having associated candidate $\widetilde{X} \rightarrow X$ with covering instructions

$$
\left(q_{11}, \ldots, q_{1 m_{1}}\right) \rightarrow p_{1}, \ldots,\left(q_{n 1}, \ldots, q_{n m_{n}}\right) \rightarrow p_{n} .
$$

For the sake of brevity we will group together our statements depending on the type of $\widetilde{X}$. In the proofs it will sometimes be convenient to carry out the steps outlined above in a different order. In particular, it is often not easy to determine beforehand when $\chi^{\text {orb }}(\widetilde{X}) / \chi^{\text {orb }}(X)$ is an integer, so this condition is imposed at the 
end. Moreover, whenever $X$ has three cone points, instead of $\chi^{\text {orb }}(\widetilde{X})=d \cdot \chi^{\text {orb }}(X)$ we will use the equivalent formula (2), expressed in terms of the data of the would-be candidate surface branched cover.

Remark 2.3. Suppose some $\widetilde{X} \stackrel{d: 1}{\rightarrow} X$ where $X$ has $n \geqslant 1$ cone points is associated

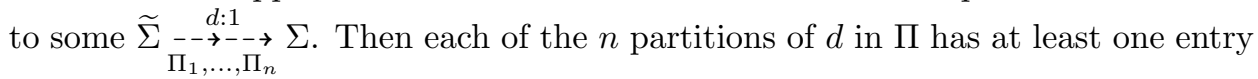

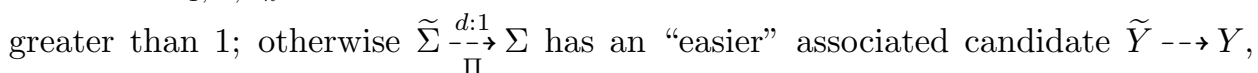
where $Y$ has less than $n$ cone points. In particular $d>1$.

Proposition 2.4. The candidate surface branched covers having associated candidate $\tilde{X} \rightarrow X$ with bad $\widetilde{X}$ are precisely those listed in (3).

Proof. We start with $\tilde{X}=S(\widetilde{p})$ for $\tilde{p} \geqslant 2$. If $X=S$ or $X=S(p)$, then $\chi^{\text {orb }}(\tilde{X}) / \chi^{\text {orb }}(X)<2$, so there is no relevant candidate.

Now suppose $X=S(p, q)$ and $\tilde{p}-\rightarrow p$, so $p=k \cdot \widetilde{p}$ for some $k$, whence $\mu_{1}=1$, $\mu_{2}=0$, and $d=k+\left(m_{1}-1\right) \cdot p=m_{2} \cdot q$. Combining these relations with $1+\frac{1}{\widetilde{p}}=$ $d \cdot\left(\frac{1}{p}+\frac{1}{q}\right)$ we get $m_{1}+m_{2}=2$, so $m_{1}=m_{2}=1$ and $k=q=d$, but $p$ is not l.c.m. $(k)$, so again there is no relevant candidate.

Turning to $X=S(2,2, p)$ we can have either $\widetilde{p} \rightarrow-\rightarrow$ or $\widetilde{p} \rightarrow-p$. In the first case we should have $\Pi_{1}=(2, \ldots, 2,1)$ and $\Pi_{2}=(2, \ldots, 2)$, which is impossible because $d$ should be both even and odd. In the second case we have $d=2 k$ and $m_{1}=m_{2}=k$, whence $m_{3}=2$, so we get item 12 in (3) in the special case where $h$ divides $2 k-h$ or conversely. Other instances of item 12 will be found below.

Now let $X=S(2,3,3)$. If $\widetilde{p}-\rightarrow 2$ (or $\widetilde{p}-\rightarrow 3)$, then $\widetilde{p}=2$ (or $\widetilde{p}=3$ ) and computing $\chi^{\text {orb }}$ we get $d=9$ (or $d=8$ ). In the first case we get item 1 in (3); in the second case we get nothing because $\Pi_{2}=(3, \ldots, 3,1)$, which is incompatible with $d=8$.

The discussion for $X=S(2,3, p)$ with $p=4,5$ is similar. We examine where $\tilde{p}$ can be mapped to, we deduce what it is (except that both 2 and 4 are possible when $\widetilde{p} \rightarrow p=4)$, in each case we determine $d$ using $\chi^{\text {orb }}$ and we check that there exist appropriate partitions of $d$. For $X=S(2,3,4)$ we get items 4 and 6 in (3), with $\widetilde{p}=2 \rightarrow 4$ in 6 , while for $X=S(2,3,5)$ we get items 9 to 11 in (3).

Let us now consider $\widetilde{X}=S(\widetilde{p}, \widetilde{q})$ with $\widetilde{p} \neq \widetilde{q}>1$ and again examine the various $X$ 's, noting first that $X$ cannot be $S$ or $S(p)$ since $d \geqslant 2$. For $X=S(p, q)$ suppose first $\widetilde{p}, \widetilde{q}-\rightarrow p$. Then $p=k \cdot \widetilde{p}=h \cdot \widetilde{q}$ and $d=k+h+\left(m_{1}-2\right) \cdot p=m_{2} \cdot q$, which we can combine with $\frac{1}{\widetilde{p}}+\frac{1}{\widetilde{q}}=d \cdot\left(\frac{1}{p}+\frac{1}{q}\right)$ easily getting $m_{1}=2$ and $m_{2}=0$, which is impossible. Now suppose $\widetilde{p} \rightarrow p$ and $\widetilde{q} \rightarrow q$, so $p=k \cdot \widetilde{p}$ and $q=h \cdot \widetilde{q}$, whence $d=k+\left(m_{1}-1\right) \cdot p=h+\left(m_{2}-1\right) \cdot q$, which leads to $m_{1}=m_{2}=1$, but then we cannot have $p=$ l.c.m. $(k)$ or $q=$ l.c.m. $(h)$, so we get nothing.

If $X=S(2,2, p)$, then we cannot have $(\widetilde{p}, \widetilde{q}) \rightarrow 2$ or $\widetilde{p} \rightarrow 2, \widetilde{q} \rightarrow 2$; otherwise $\widetilde{X}$ would be good. If $\widetilde{p} \rightarrow-\rightarrow 2$ and $\widetilde{q} \rightarrow-\rightarrow$, then $d$ should be both even and odd, which is impossible. So $(\widetilde{p}, \widetilde{q})-\rightarrow p, d=2 k, m_{1}=m_{2}=k, m_{3}=2$ and we get the last item in (3) with $h$ and $2 k-h$ not multiples of each other.

For $X=S(2,3, p)$, considering where $\widetilde{p}$ and $\widetilde{q}$ can be mapped, we again see what they can be, we determine $d$ using $\chi^{\text {orb }}$, and we check that three appropriate partitions exist, getting nothing for $p=3$, items 2 and 3 in (3) for $p=4$, and items 5,7 , and 8 for $p=5$, which completes the proof. 
We omit the straightforward proof of the next result:

Proposition 2.5. The candidate surface branched covers having associated candidate $S \rightarrow X$ are

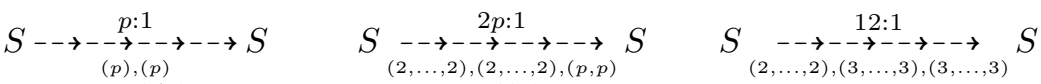

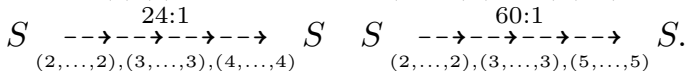

Proposition 2.6. The candidate surface branched covers having associated candidate $S(\widetilde{p}, \widetilde{p}) \rightarrow X$ with $\widetilde{p}>1$ are

$$
\begin{aligned}
& \begin{array}{c}
4: 1 \\
S-\rightarrow-\rightarrow-\rightarrow--\rightarrow--\rightarrow
\end{array} \\
& \begin{array}{c}
8: 1 \\
S \underset{(2, \ldots, 2),(3,3,1,1),(4,4)}{--\rightarrow--\rightarrow-\rightarrow} S
\end{array}
\end{aligned}
$$

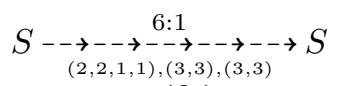

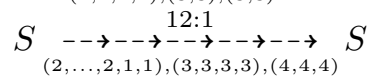

$$
\begin{aligned}
& S \rightarrow \underset{12: 1}{ } \rightarrow-\rightarrow-\rightarrow-\rightarrow \\
& S \quad 2 k+1: 1
\end{aligned}
$$

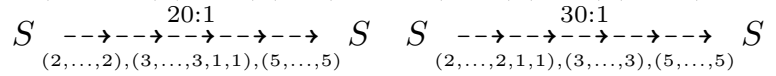

$$
\begin{aligned}
& S \underset{(2, \ldots, 2,1),(2, \ldots, 2,1),(2 k+1)}{-\rightarrow-\rightarrow--\rightarrow--\rightarrow--\rightarrow--\rightarrow}
\end{aligned}
$$

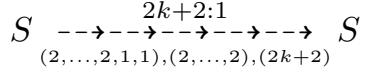

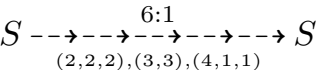

$$
\begin{aligned}
& \begin{array}{c}
12: 1 \\
\substack{(2, \ldots, 2),(3, \ldots, 3),(4,4,2,2)}
\end{array}
\end{aligned}
$$

with arbitrary $k \geqslant 1$ in the last two items.

Proof. Since $\chi^{\operatorname{orb}}(S(\tilde{p}, \tilde{p}))=\frac{2}{\widetilde{p}} \leqslant 1$ and $d \geqslant 2$ we cannot have $X=S$ or $X=S(p)$. Suppose then $X=S(p, q)$, so $\frac{2}{\widetilde{p}}=\frac{d}{p}+\frac{d}{q}$. If $\tilde{p}, \tilde{p} \rightarrow p$, then $p=k \cdot \widetilde{p}$ and $d=$ $2 k+\left(m_{1}-2\right) \cdot p=m_{2} \cdot q$, whence $m_{1}=2$ and $m_{2}=0$, which is absurd. If $\tilde{p}--\rightarrow p$ and $\widetilde{p} \rightarrow q$, then $p=k \cdot \widetilde{p}$ and $q=h \cdot \widetilde{p}$, whence $d=k+\left(m_{1}-1\right) \cdot p=h+\left(m_{2}-1\right) \cdot q$, which gives $m_{1}=m_{2}=1$, but then we cannot have $p=$ l.c.m. $(k)$ or $q=$ l.c.m. $(h)$, so we get nothing.

Assume now $X=S(2,2, p)$. If $(\widetilde{p}, \widetilde{p}) \rightarrow 2$, then $\widetilde{p}=2$ and $p=d=2 k+2$, which leads to the last item in (5). If $\widetilde{p} \rightarrow-\rightarrow 2$ and $\tilde{p} \rightarrow-\rightarrow$, then $\tilde{p}=2$ and $p=d=2 k+1$, so we get the penultimate item in (5). Of course we cannot have $\widetilde{p}-\rightarrow 2$ and $\widetilde{p}-\rightarrow p$; otherwise $d$ should be both even and odd. If $(\widetilde{p}, \widetilde{p})-\rightarrow p$, then $p=k \cdot \widetilde{p}$ and $d=2 k$, so $\Pi_{3}=(k, k)$, but then $p \neq$ l.c.m. $(k, k)$.

If $X=S(2,3,3)$, then $d \cdot \widetilde{p}=12$. Of course we cannot have $\widetilde{p}--\rightarrow 2$ and $\widetilde{p}--\rightarrow 3$. If $(\tilde{p}, \tilde{p}) \rightarrow 2$, then $\widetilde{p}=2$ and $d=6$, so we get item 2 in (15), while if $(\widetilde{p}, \widetilde{p})-\rightarrow 3$, then $\widetilde{p}=3$ and $d=4$, which is impossible since there would be a partition of 4 consisting of 3's only. For $\widetilde{p}-\rightarrow 3$ and $\widetilde{p}-\rightarrow 3$ again $\widetilde{p}=3$ and $d=4$, whence item 1 in (5).

The discussion for $X=S(2,3, p)$ with $p=4,5$ is similar. We get items 3 to 6 in (5) for $p=4$ and items 7 to 9 for $p=5$.

Proposition 2.7. The candidate surface branched covers having associated candidate $S(2,2, \widetilde{p}) \rightarrow X$ with $\tilde{p}>1$ are

$$
\begin{aligned}
& \begin{array}{c}
4: 1 \\
S-\rightarrow-\rightarrow-\rightarrow--\rightarrow--\rightarrow
\end{array} \\
& S-\rightarrow-\rightarrow-1 \rightarrow--\rightarrow-\rightarrow S
\end{aligned}
$$

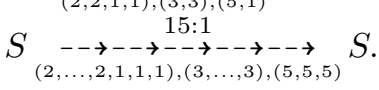

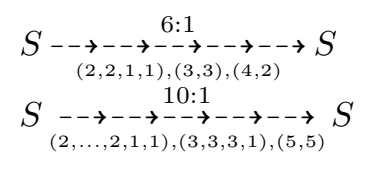


Proof. Note first that $X$ cannot be $S$ or $S(p)$ since $d \geqslant 2$. If $X=S(p, q)$, we have the following possibilities:

- $(2,2, \widetilde{p})-\rightarrow p$. Then $p=2 k$, so $d=k+k+2 k / \widetilde{p}+\left(m_{1}-3\right) 2 k=m_{2} q$.

- $\widetilde{p} \rightarrow p$ and $(2,2) \rightarrow q$. Then $p=k \widetilde{p}$ and $q=2 h$, so $d=k+\left(m_{1}-1\right) k \widetilde{p}=$ $h+h+\left(m_{2}-2\right) 2 h$.

- $(2, \widetilde{p}) \rightarrow p$ and $2 \rightarrow q$. Then $p=2 k$ and $q=2 h$, so $d=k+2 k / \widetilde{p}+$ $\left(m_{1}-2\right) 2 k=h+\left(m_{2}-1\right) 2 h$.

Since $\frac{1}{\widetilde{p}}=\frac{d}{p}+\frac{d}{q}$, in all cases we deduce that $m_{1}+m_{2}=2$, which is absurd, so we do not get any candidate cover.

Now suppose $X=S(2,2, p)$. We have the following possibilities:

$$
\begin{array}{lll}
(2,2, \widetilde{p}) \rightarrow-\rightarrow 2 & (2,2, \widetilde{p}) \rightarrow-\rightarrow p & \\
(2,2)-\rightarrow 2, \widetilde{p}-\rightarrow 2 & (2,2)-\rightarrow 2, \widetilde{p}-\rightarrow p & \widetilde{p}-\rightarrow 2,(2,2)-\rightarrow p \\
(2, \widetilde{p})-\rightarrow 2,2-\rightarrow 2 & (2, \widetilde{p})-\rightarrow 2,2-\rightarrow p & 2-\rightarrow 2,(2, \widetilde{p})-\rightarrow p, \\
2-\rightarrow 2,2 \rightarrow-\rightarrow 2, \widetilde{p}-\rightarrow p & 2-\rightarrow 2, \widetilde{p}-\rightarrow 2,2-\rightarrow p &
\end{array}
$$

and $\widetilde{p}$ must actually be 2 in items $1,3,5,6,7$ and 10 . Items $1,3,5,6$ and 8 are then impossible because $d$ should be both even and odd. In item 2 we have $d=2 k$ and $m_{1}=m_{2}=k$, whence $m_{3}=2$, which is impossible. In items 4 and 7 we have $d=2 k$, whence $m_{1}=k+1$ and $m_{2}=k$, so $m_{3}=1$, which is absurd. In items 9 and 10 we have $d=2 k+1$ and $m_{1}=m_{2}=k+1$, whence $m_{3}=1$, which is absurd. This shows that there is no candidate cover for $X=S(2,2, p)$.

The cases where $X=S(2,3, p)$ for $p=3,4,5$ are easier to discuss and hence left to the reader. For $p=3$ there is nothing, for $p=4$ there are items 1 and 2 in ([6), and for $p=5$ items 3 to 5 .

The candidate surface branched covers having associated candidate covers $S(2,3, \widetilde{p}) \rightarrow X$ for $\widetilde{p}=3,4,5$ are not hard to analyze using the same methods employed above, so we will not spell out the proofs of the next two results. The most delicate point is always to exclude the cases $X=S(p, q)$ and $X=S(2,2, p)$.

Proposition 2.8. The only candidate surface branched cover having associated candidate $S(2,3,3) \rightarrow X$ is $S \underset{\substack{-\rightarrow-\rightarrow-\rightarrow \rightarrow-\rightarrow \\(2,2,1),(3,1,1),(5)}}{5: 1}$.

Proposition 2.9. There are no candidate surface branched covers having associated candidate of the form $S(2,3,4) \rightarrow X$ or $S(2,3,5) \rightarrow X$.

Spherical structures. As already mentioned, any good 2-orbifold $X$ with $\chi^{\text {orb }}(X)>0$ has a spherical structure, given by the action of some finite group $\Gamma$ of isometries on the metric sphere $\mathbb{S}$. We will now explicitly describe each relevant $\Gamma$, thus identifying $X$ with the quotient $\mathbb{S} / \Gamma$. To this end we will always regard $\mathbb{S}$ as the unit sphere of $\mathbb{C} \times \mathbb{R}$.

The football. The geometry of $S(p, p)$ is very easy, even if for consistency with what follows we will not give the easiest description. Consider in $\mathbb{S}$ a wedge with vertices at the poles $(0, \pm 1)$ and edges passing through $(1,0)$ and $\left(\mathrm{e}^{i \pi / p}, 0\right)$, so the width is $\pi / p$. Now define $\widetilde{\Gamma}_{(p, p)}$ as the group of isometries of $\mathbb{S}$ generated by the reflections in the edges of the wedge, and denote by $\Gamma_{(p, p)}$ its subgroup of orientation-preserving isometries. Then $\Gamma_{(p, p)}$ is generated by the rotation of angle $2 \pi / p$ around the poles $(0, \pm 1)$, a fundamental domain for $\Gamma_{(p, p)}$ is the union of any two wedges sharing an edge, and $S(p, p)=\mathbb{S} / \Gamma_{(p, p)}$. See Fig. 1-left. 

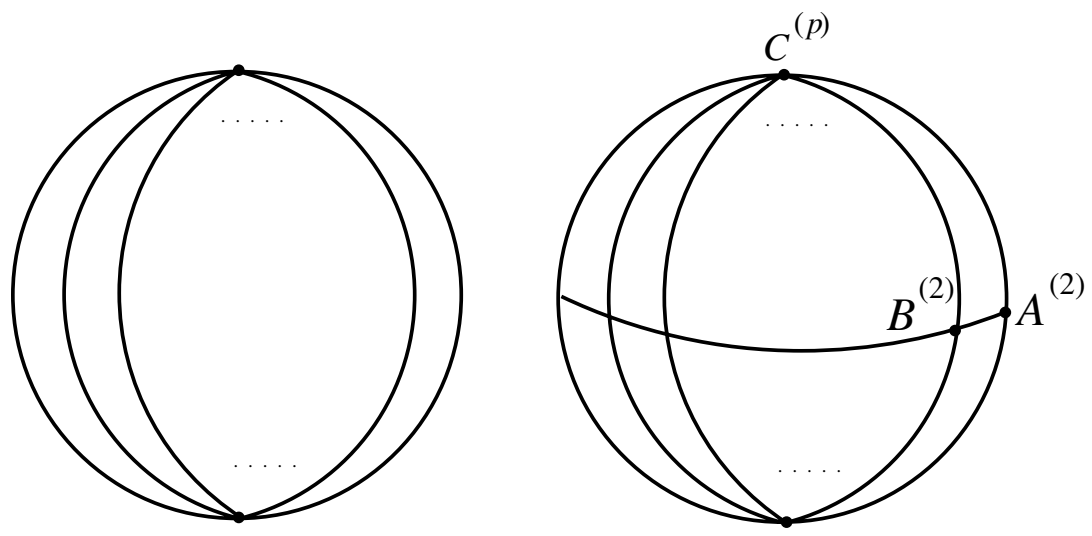

FIgURE 1. Tessellations of $\mathbb{S}$ by fundamental domains of $\widetilde{\Gamma}_{(p, p)}$ and $\widetilde{\Gamma}_{(2,2, p)}$
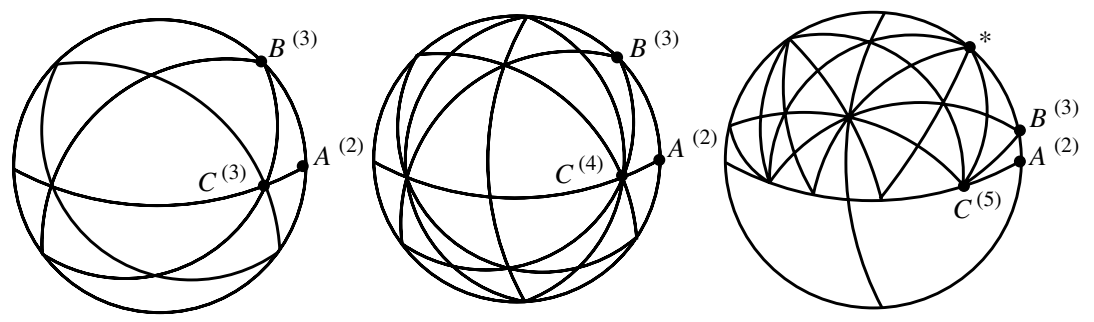

Figure 2. Tessellations of $\mathbb{S}$ by triangular fundamental domains of $\widetilde{\Gamma}_{(2,3,3)}, \widetilde{\Gamma}_{(2,3,4)}$, and (partial) $\widetilde{\Gamma}_{(2,3,5)}$

Triangular orbifolds. The remaining spherical 2-orbifolds $S(2, q, p)$ with either $q=$ 2 and $p \geqslant 2 j$ or $q=3$ and $p=3,4,5$ are called triangular. The corresponding group $\Gamma_{(2, q, p)}$ is the subgroup of orientation-preserving elements of a group $\widetilde{\Gamma}_{(2, q, p)}$ generated by the reflections in the edges of a triangle $\Delta_{(2, q, p)}$ with angles $\pi / 2, \pi / q, \pi / p$. A fundamental domain of $\Gamma_{(2, q, p)}$ is then the union of $\Delta_{(2, q, p)}$ with its image under any of the reflections in its edges, and $\Gamma_{(2, q, p)}=\left\langle\alpha, \beta, \gamma \mid \alpha^{2}=\beta^{q}=\gamma^{p}=\alpha \cdot \beta \cdot \gamma=1\right\rangle$ where $\alpha, \beta, \gamma$ are rotations centered at the vertices of $\Delta_{(2, q, p)}$.

The main point here is of course that the triangles $\Delta_{(2, q, p)}$ exist in $\mathbb{S}$. The choice for $q=2$ and arbitrary $p$ is easy: $A=(1,0), B=\left(\mathrm{e}^{i \pi / p}, 0\right)$ and $C=(0,1)$; see Fig. 1 1-right. For $q=3$ see Fig. [2]

In each case the pictures also show the images of $\Delta(2, q, p)$ under the action of $\widetilde{\Gamma}_{(2, q, p)}$. A fundamental domain for the group $\Gamma_{(2, q, p)}$ giving $S(2, q, p)$ is always the union of any two triangles sharing an edge. In all the pictures for $q=3$ we have $A=(1,0)$. Moreover for $p=3$ we have $B=\left(\sqrt{\frac{1}{3}}, \sqrt{\frac{2}{3}}\right)$ and $C=\left(\sqrt{\frac{1}{3}}+i \sqrt{\frac{2}{3}}, 0\right)$, while for $p=4$ we have $B=\left(\sqrt{\frac{2}{3}}, \sqrt{\frac{1}{3}}\right)$ and $C=\left(\sqrt{\frac{1}{2}}+i \sqrt{\frac{1}{2}}, 0\right)$; for $p=5$ the exact values of the coordinates of $B$ and $C$ are more complicated. 
Remark 2.10. Each group $\widetilde{\Gamma}_{(2,3, p)}$ is the symmetry group of a Platonic solid inscribed in $\mathbb{S}$ : the tetrahedron for $p=3$, the octahedron (or its dual cube) for $p=4$, and the dodecahedron (or its dual icosahedron) for $p=5$.

Geometric realizations. Let a candidate orbifold cover $\tilde{X} \rightarrow X$ with covering instructions be associated to some candidate surface branched cover. When $\widetilde{X}$ is bad and $X$ is spherical, Lemma 1.1 implies that $\widetilde{X} \rightarrow X$ is not realizable. The results established above show that $X$ is never bad, so we are left to show that the cover is realizable when both $\tilde{X}$ and $X$ are spherical. The case $\widetilde{X}=S$ (Proposition 2.5) is however easy, since a realization of the candidate covers in (4) is given precisely by the geometric universal cover $\mathbb{S} \rightarrow X$ we have fixed. We will now deal with all the other cases, by constructing an isometry $\widetilde{f}: \mathbb{S} \rightarrow \mathbb{S}$ that induces a realization $f: \widetilde{X} \rightarrow X$ such that $\pi \circ \widetilde{f}=f \circ \widetilde{\pi}$.

Proposition 2.11. All the candidate surface branched covers of Proposition 2.6 are realizable.

Proof. The following list describes the candidate orbifold covers $\widetilde{X} \rightarrow X$ and the covering instructions associated to the items in (5), together with the corresponding isometry $\widetilde{f}: \mathbb{S} \rightarrow \mathbb{S}$ inducing the desired $f$ via the geometric universal covers $\widetilde{\pi}: \mathbb{S} \rightarrow \widetilde{X}$ and $\pi: \mathbb{S} \rightarrow X$ fixed above:

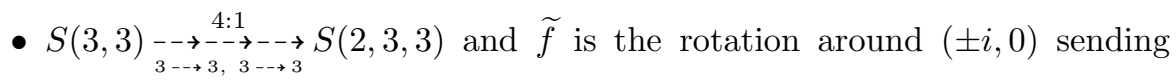
$(0,1)$ to point $B$ of Fig. [2-left;

- $S(2,2) \underset{\substack{6: \rightarrow-\rightarrow-\rightarrow \\(2,2) \rightarrow 2}}{6: \rightarrow 2}(2,3,3)$ and $\tilde{f}=\operatorname{rot}_{( \pm i, 0)}(\pi / 2)$;

- $S(4,4) \underset{\substack{6: 1 \\(4,4) \rightarrow-\rightarrow 4}}{\substack{\rightarrow-\rightarrow \rightarrow \\ \text { and }}} S(2,3,4)$ and $\tilde{f}$ is the identity;

- $S(3,3) \underset{\substack{8: \rightarrow-\rightarrow \\(3,3) \rightarrow \rightarrow 3}}{\rightarrow} S(2,3,4)$ and $\tilde{f}$ is the rotation around $( \pm i, 0)$ sending $(0,1)$ to point $B$ of Fig. 2r-center;

- $S(2,2) \underset{\substack{12 \rightarrow-\rightarrow \rightarrow \\(2,2) \rightarrow 2}}{12: 1} S(2,3,4)$ and $\tilde{f}=\operatorname{rot}_{( \pm i, 0)}(\pi / 2)$;

- $S(2,2) \underset{\substack{12 \rightarrow-1 \\(2,2) \rightarrow 4}}{12 \rightarrow \rightarrow}(2,3,4)$ and $\tilde{f}$ is the identity;

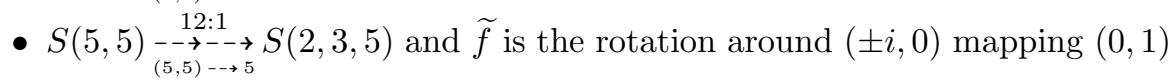
to the point labelled $*$ in Fig. 2,

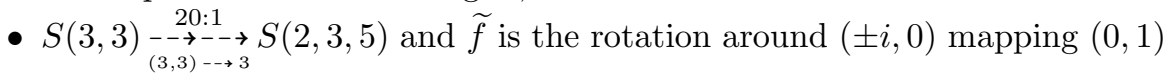
to point $B$ in Fig. 2-right;

- $S(2,2) \underset{\substack{3 \rightarrow-\rightarrow-\rightarrow \\(2,2) \rightarrow \rightarrow 2}}{30: 1}(2,3,5)$ and $\widetilde{f}$ is the identity;

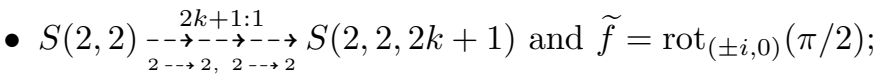

- $S(2,2) \underset{\substack{2 k+2:-\rightarrow \rightarrow \\(2,2) \rightarrow 2}}{\substack{2 k \rightarrow 2}}(2,2,2 k+2)$ and $\tilde{f}=\operatorname{rot}_{( \pm i, 0)}(\pi / 2)$.

The proof is complete.

Proposition 2.12. All the candidate surface branched covers of Proposition 2.7 are realizable. 
Proof. The candidate orbifold covers with covering instructions associated to the items in (6), and the corresponding isometries $\widetilde{f}: \mathbb{S} \rightarrow \mathbb{S}$, are as follows:

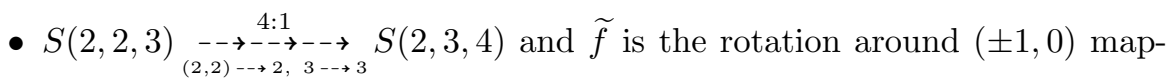
ping $(0,1)$ to point $B$ in Fig. 2-center followed by a rotation of angle $\pi / 6$ around $\pm B$

- $S(2,2,2) \underset{\substack{-\rightarrow \rightarrow-\rightarrow \rightarrow-\rightarrow \\(2,2) \rightarrow 2,2 \rightarrow 4}}{6: 1} \mathfrak{S}(2,3,4)$ and $\widetilde{f}$ is the identity;

- $S(2,2,5) \underset{\substack{-\rightarrow \rightarrow-\rightarrow \rightarrow-\rightarrow \\(2,2) \rightarrow 2,5 \rightarrow 5}}{6: \rightarrow} \rightarrow(2,3,5)$ and $\tilde{f}$ is the rotation around $( \pm 1,0)$, sending $(0,1)$ to the point of order 5 best visible in the upper hemisphere in Fig. 2-right;

- $S(2,2,3) \underset{\substack{-\rightarrow \rightarrow-\rightarrow \rightarrow-\rightarrow \rightarrow \\(2,2) \rightarrow 2,3 \rightarrow \rightarrow 3}}{10: 1} S(2,3,5)$ and $\tilde{f}$ is the rotation around $( \pm i, 0)$ that maps the pole $(0,1)$ to point $B$ in Fig. 2-right followed by a rotation of angle $\pi / 6$ around $\pm B$;

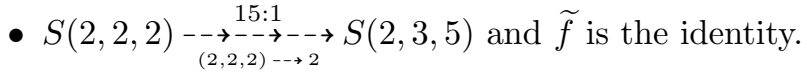

The proof is complete.

Proposition 2.13. The candidate surface branched cover of Proposition 2.8 is realizable.

Proof. The candidate orbifold cover is in this case

$$
S(2,3,3) \underset{\substack{-\rightarrow \rightarrow-\rightarrow \rightarrow-\rightarrow \\ 2 \rightarrow 2,(3,3) \rightarrow 3}}{5: \rightarrow}(2,3,5)
$$

and the isometry $\tilde{f}: \mathbb{S} \rightarrow \mathbb{S}$ inducing its realization is the rotation of angle $\pi / 4$ around $( \pm 1,0)$.

As already mentioned, color pictures of the covers just described are available on the web 20]. We will illustrate here only the isometries $\widetilde{f}: \mathbb{S} \rightarrow \mathbb{S}$ realizing $S(5,5) \rightarrow S(2,3,5)$ and $S(2,3,3) \rightarrow S(2,3,5)$. Fig. 3-left shows (marked by a thicker line) a partial image of the tessellation for $\widetilde{\Gamma}_{(5,5)}$ as in Fig. 1 1 left on the sphere with the tessellation for $\widetilde{\Gamma}_{(2,3,5)}$ as in Fig. 2-right. Fig. 3-right does the same, after a stereographic projection, with the image of the tessellation for $\widetilde{\Gamma}_{(2,3,3)}$ as in Fig. 2-left and the tessellation for $\widetilde{\Gamma}_{(2,3,5)}$ as in Fig. 2-right. Note that in Fig. 3-left every thick line covers a thin line, but the same is false in Fig. 3-right.

Algebraic realizations. We recall here [10, 18, that a realization of a candidate surface branched cover

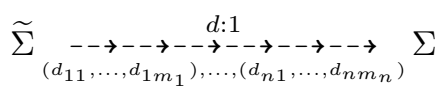

corresponds to the choice of permutations $\sigma_{1}, \ldots, \sigma_{n} \in \mathfrak{S}_{d}$ such that:

- $\sigma_{i}$ has cycles of lengths $\left(d_{i j}\right)_{j=1}^{m_{i}}$;

- the product $\sigma_{1} \cdots \sigma_{n}$ is the identity;

- the subgroup of $\mathfrak{S}_{d}$ generated by $\sigma_{1}, \ldots, \sigma_{n}$ acts transitively on $\{1, \ldots, d\}$. 

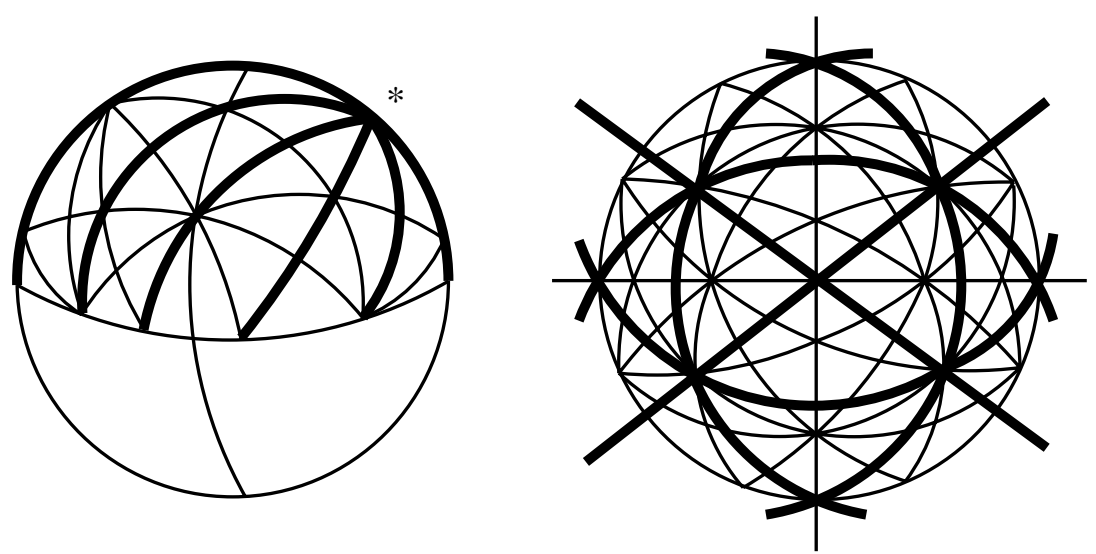

Figure 3. Two samples of coverings: $S(5,5) \rightarrow S(2,3,5)$, and $S(2,3,3) \rightarrow S(2,3,5)$

Using this fact we will now give an alternative proof of the fact that when the Euler characteristic is positive the candidate covers not listed as exceptional in Theorem 2.1 are realizable. The verification is by direct inspection: we denote by $\left(i_{0}, \ldots, i_{p-1}\right)$ the cycle mapping $i_{j}$ to $i_{j+1}(\bmod p)$ and we multiply permutations as functions, so $(1,2) \cdot(2,3)=(1,2,3)$.

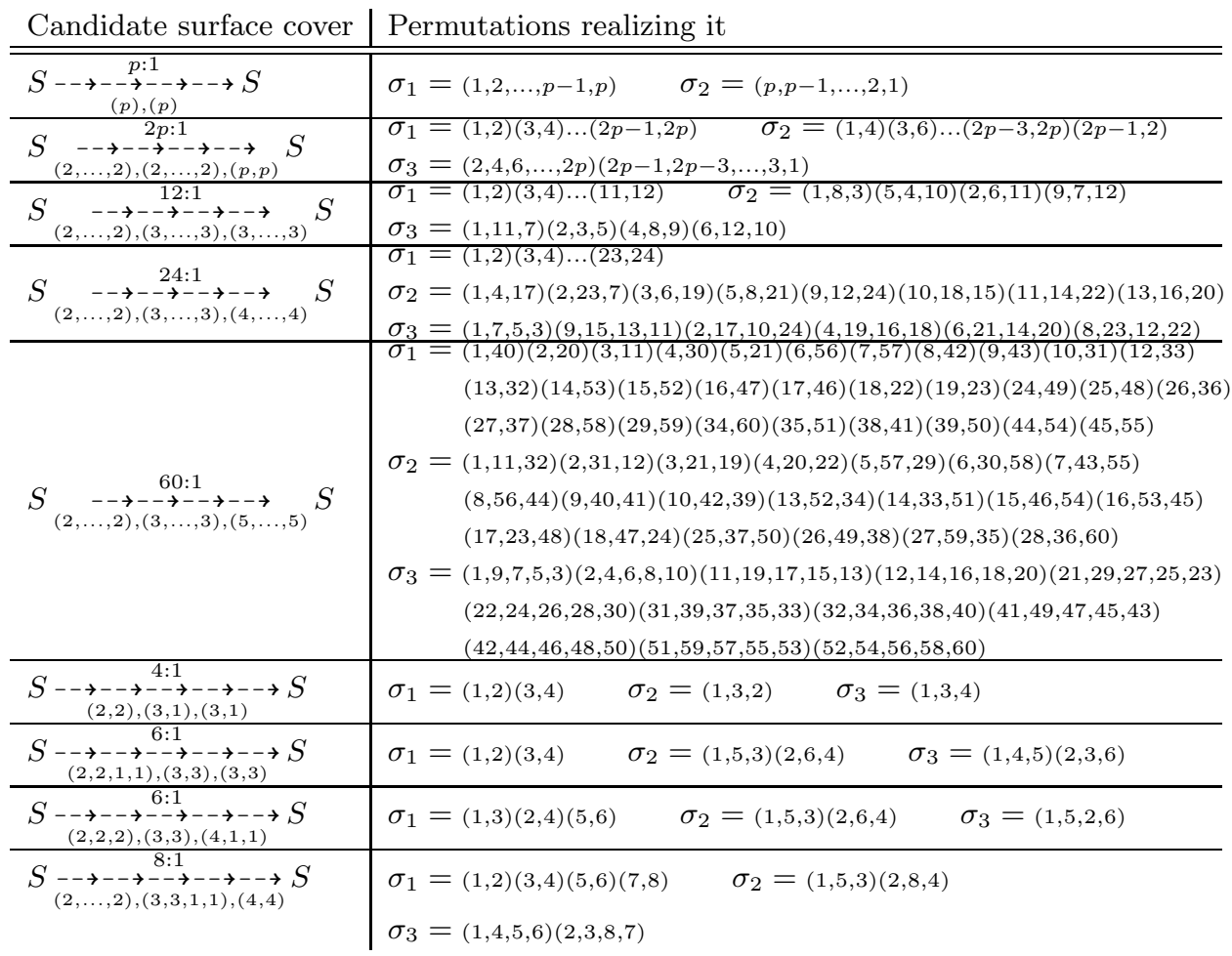




\begin{tabular}{|c|c|}
\hline$S_{(2, \ldots, 2,1,1),(3,3,3,3),(4,4,4)}^{-\cdots \rightarrow--\rightarrow--\rightarrow--\rightarrow--\rightarrow} S$ & $\begin{array}{l}\sigma_{1}=(1,12)(2,8)(9,10)(6,7)(3,4) \\
\sigma_{2}=(3,2,1)(6,5,4)(9,8,7)(12,11,10) \\
\sigma_{3}=(1,10,7,4)(2,9,11,12)(3,5,6,8)\end{array}$ \\
\hline 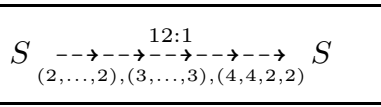 & $\begin{array}{l}\sigma_{1}=(1,12)(8,11)(2,5)(9,10)(6,7)(3,4) \\
\sigma_{2}=(3,2,1)(6,5,4)(9,8,7)(12,11,10) \\
\sigma_{3}=(1,10,7,4)(2,6,8,12)(3,5)(9,11)\end{array}$ \\
\hline$S \underset{(2, \ldots, 2),(3, \ldots, 3),(5,5,1,1)}{\substack{12: 1 \\
-}} S$ & $\begin{array}{l}\sigma_{1}=(2,5)(1,4)(6,12)(3,9)(7,8)(10,11) \\
\sigma_{2}=(3,2,1)(6,5,4)(9,8,7)(12,11,10) \\
\sigma_{3}=(1,5,3,7,9)(2,6,10,12,4)\end{array}$ \\
\hline 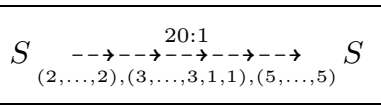 & $\begin{array}{l}\sigma_{1}=(6,19)(14,20)(2,4)(3,9)(5,7)(8,10)(12,13)(15,16)(11,17)(1,18) \\
\sigma_{2}=(3,2,1)(6,5,4)(9,8,7)(12,11,10)(15,14,13)(18,17,16) \\
\sigma_{3}=(1,16,13,10,9)(2,5,8,11,18)(3,7,6,19,4)(12,14,20,15,17)\end{array}$ \\
\hline$S \underset{\substack {3, \ldots, 2,1,1),(3, \ldots, 3),(5, \ldots, 5) \\
\begin{subarray}{c}{-\rightarrow--\rightarrow--\rightarrow--\rightarrow--\rightarrow{ 3 , \ldots , 2 , 1 , 1 ) , ( 3 , \ldots , 3 ) , ( 5 , \ldots , 5 ) \\
\begin{subarray} { c } { - \rightarrow - - \rightarrow - - \rightarrow - - \rightarrow - - \rightarrow } }\end{subarray}}{S}$ & $\begin{aligned} \sigma_{1}= & (1,14)(2,4)(3,26)(5,7)(6,30)(8,10)(9,24) \\
& (11,13)(12,20)(15,16)(17,22)(18,19)(23,25)(27,28) \\
\sigma_{2}= & (3,2,1)(6,5,4)(9,8,7)(12,11,10)(15,14,13) \\
& (18,17,16)(21,10,19)(24,23,22)(27,26,25)(30,29,28) \\
\sigma_{3}= & (1,15,17,23,26)(2,5,8,11,14)(3,27,29,30,4) \\
& (6,28,25,24,7)(9,22,18,20,10)(12,21,19,16,13)\end{aligned}$ \\
\hline $\begin{array}{c}2 k+1: 1 \\
S \begin{array}{c}--\rightarrow--\rightarrow--\rightarrow--\rightarrow--\rightarrow \\
(2, \ldots, 2,1),(2, \ldots, 2,1),(2 k+1)\end{array}\end{array}$ & $\begin{array}{l}\sigma_{1}=(1,2)(3,4) \cdots(2 k-1,2 k) \quad \sigma_{2}=(2,3)(4,5) \cdots(2 k, 2 k+1) \\
\sigma_{3}=(1,3,5,7, \ldots, 2 k-1,2 k+1,2 k, 2 k-2, \ldots, 6,4,2)\end{array}$ \\
\hline 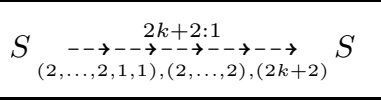 & $\begin{array}{l}\sigma_{1}=(1,2)(3,4) \cdots(2 k-1,2 k) \\
\sigma_{2}=(2,3)(4,5) \cdots(2 k, 2 k+1)(2 k+2,1) \\
\sigma_{3}=(1,3,5,7, \ldots, 2 k-1,2 k+1,2 k, 2 k-2, \ldots, 6,4,2,2 k+2)\end{array}$ \\
\hline $\begin{array}{c}S: 1 \\
S-\rightarrow--\rightarrow--\rightarrow--\rightarrow--\rightarrow S \\
(2,1,1),(3,1),(4)\end{array}$ & $\sigma_{1}=(1,2) \quad \sigma_{2}=(1,3,4) \quad \sigma_{3}=(1,2,4,3)$ \\
\hline $\begin{array}{c}6: 1 \\
S-\underset{(2,2,1,1),(3,3),(4,2)}{ }\end{array}$ & $\sigma_{1}=(1,2)(3,4) \quad \sigma_{2}=(1,3,5)(2,6,4) \quad \sigma_{3}=(1,4)(2,5,3,6)$ \\
\hline $\begin{array}{c}6: 1 \\
S \underset{(2,2,1,1),(3,3),(5,1)}{ }\end{array}$ & $\sigma_{1}=(1,2)(3,4) \quad \sigma_{2}=(1,3,2)(4,6,5) \quad \sigma_{3}=(1,3,5,6,4)$ \\
\hline 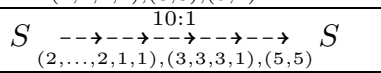 & $\begin{array}{l}\sigma_{1}=(2,8)(1,4)(6,7)(9,10) \quad \sigma_{2}=(3,2,1)(6,5,4)(9,8,7) \\
\sigma_{3}=(1,5,6,8,3)(2,9,10,7,4)\end{array}$ \\
\hline$S \underset{(2, \ldots, 2,1,1,1),(3, \ldots, 3),(5,5,5)}{\substack{15: 1\\
}} S$ & $\begin{array}{l}\sigma_{1}=(2,11)(12,13)(9,10)(5,7)(3,4)(6,14) \\
\sigma_{2}=(3,2,1)(6,5,4)(9,8,7)(12,11,10)(15,14,13) \\
\sigma_{3}=(1,2,12,14,4)(3,5,8,9,11)(6,15,13,10,7)\end{array}$ \\
\hline$S \underset{\substack{(2,2,1),(3,1,1),(5) \\
-\rightarrow--\rightarrow--\rightarrow}}{5: 1} S$ & $\sigma_{1}=(1,2)(3,4) \quad \sigma_{2}=(5,3,1) \quad \sigma_{3}=(1,2,3,4,5)$ \\
\hline
\end{tabular}

\section{The Euclidean case}

In this section we investigate the realizability of candidate surface branched covers having associated candidate orbifold covers $\widetilde{X}-\rightarrow X$ with $\chi^{\text {orb }}(\widetilde{X})=\chi^{\text {orb }}(X)=0$. This means that $\widetilde{X}$ and $X$ must belong to the list

$$
T, \quad S(2,4,4), \quad S(2,3,6), \quad S(3,3,3), \quad S(2,2,2,2),
$$

where $T$ is the torus. Recalling that the orders of the cone points of $\widetilde{X}$ must divide

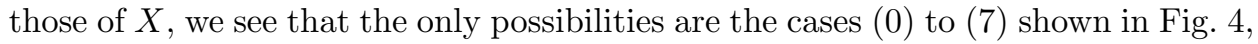
that we will analyze using Euclidean geometry. Namely:

- We will fix on $X$ a Euclidean structure given by some $\pi: \mathbb{E} \rightarrow X$.

- We will assume that $\widetilde{X} \stackrel{d: 1}{\rightarrow-\rightarrow}$ is realized by some map $f$, we will use Lemma 1.3 to deduce there is a corresponding affine map $\tilde{f}: \mathbb{E} \rightarrow \mathbb{E}$, and we will analyze $\tilde{f}$ to show that $d$ must satisfy certain conditions.

- We will employ the calculations of the previous point to show that if $d$ satisfies the conditions, then $\widetilde{f}$, whence $f$, exists.

Remark 3.1. It would be possible but rather complicated to exhibit permutations realizing the candidate covers shown to exist by geometric methods, in the spirit of the last part of the previous section. Permutations corresponding to Theorem 0.4 
(1)

(2)
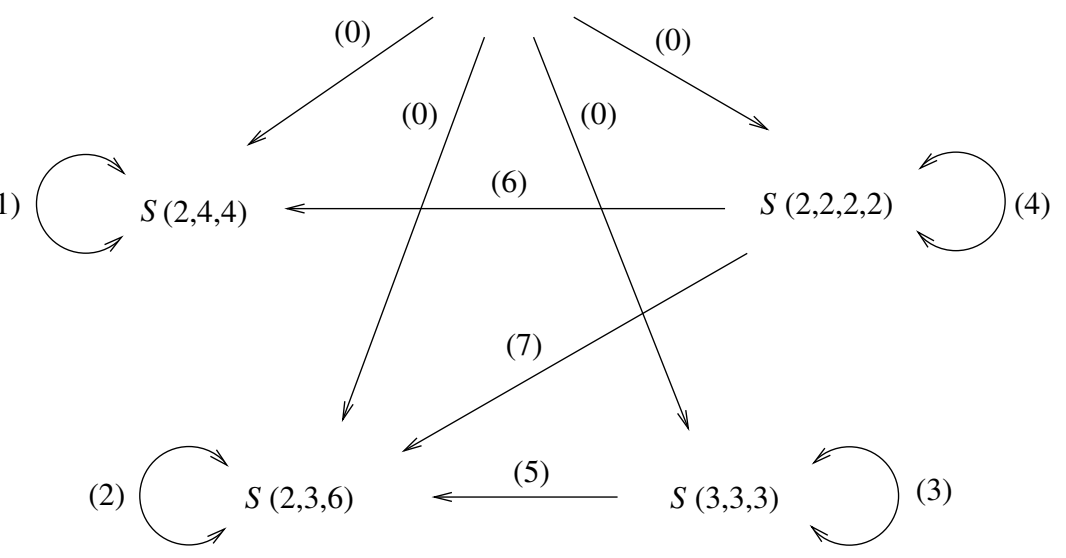

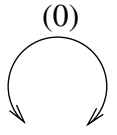

T

Figure 4. Possible covers between Euclidean orbifolds
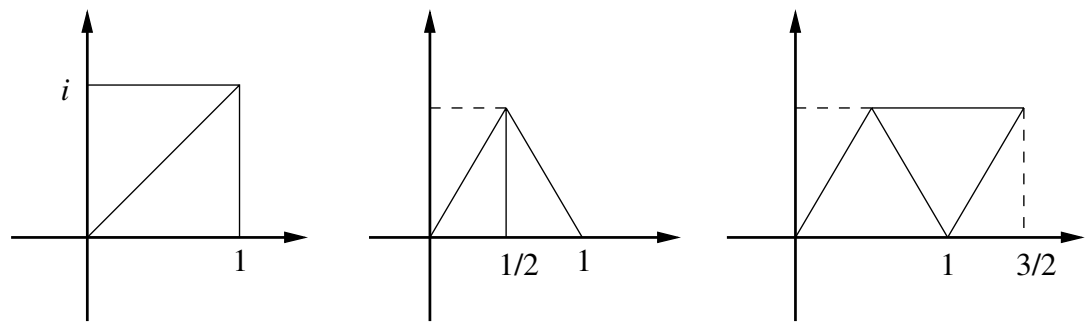

Figure 5. The fixed fundamental domains for $S(2,4,4), S(2,3,6)$ and $S(3,3,3)$

together with algebraic interpretations of the results of the present paper and a discussion of the relations with previous results [25, 26, 27, 9] are provided in [19.

Euclidean structures. On each of the three triangular orbifolds $S(p, q, r)$ with $\frac{1}{p}+$ $\frac{1}{q}+\frac{1}{r}=1$ the Euclidean structure (unique up to rescaling) is constructed essentially as in the spherical case. We take a triangle $\Delta(p, q, r)$ in $\mathbb{E}$ with angles $\pi / p, \pi / q, \pi / r$, the group $\widetilde{\Gamma}_{(p, q, r)}$ generated by the reflections in the edges of $\Delta(p, q, r)$, and its subgroup $\Gamma_{(p, q, r)}$ of orientation-preserving isometries. Then $S(p, q, r)$ is the quotient of $\mathbb{E}$ under the action of $\Gamma_{(p, q, r)}$, which is generated by the rotations of angles $2 \pi / p, 2 \pi / q, 2 \pi / r$ around the vertices of $\Delta(p, q, r)$, and a fundamental domain is given by the union of $\Delta(p, q, r)$ with any of its reflected copies in one of the edges. In each case we now make a precise choice for the vertices $\widetilde{A}^{(p)}, \widetilde{B}^{(q)}, \widetilde{C}^{(r)}$ of $\Delta(p, q, r)$ and determine the resulting area $\mathcal{A}$ of $S(p, q, r)$. See also Fig. 5 . 

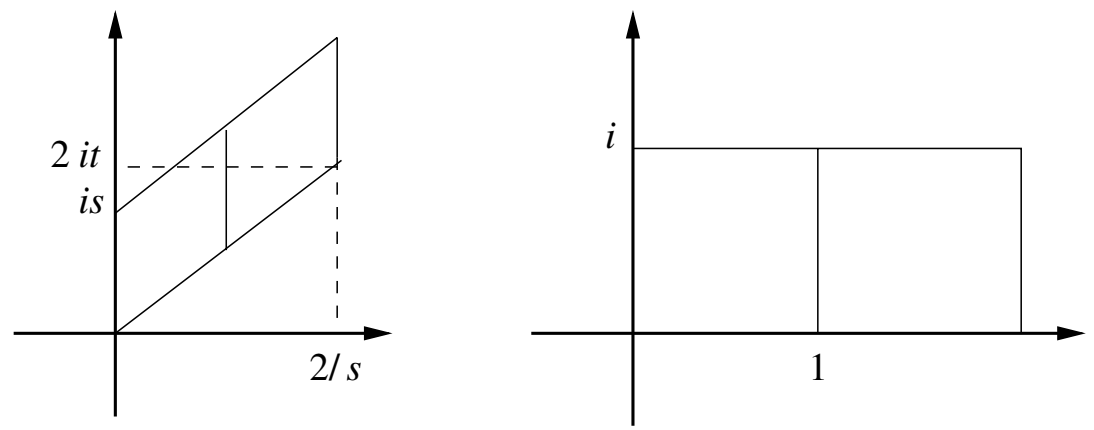

Figure 6. The fundamental domains for $S(2,2,2,2)$ for general $s, t$ and for $s=1, t=0$

$$
\begin{array}{lllll}
\Delta(2,4,4): & \widetilde{A}^{(2)}=1, & \widetilde{B}^{(4)}=0, & \widetilde{C}^{(4)}=1+i, & \mathcal{A}(S(2,4,4))=1, \\
\Delta(2,3,6): & \widetilde{A}^{(2)}=\frac{1}{2}, & \widetilde{B}^{(3)}=0, & \widetilde{C}^{(6)}=\frac{1+i \sqrt{3}}{2}, & \mathcal{A}(S(2,3,6))=\frac{\sqrt{3}}{4}, \\
\Delta(3,3,3): & \widetilde{A}^{(3)}=0, & \widetilde{B}^{(3)}=1, & \widetilde{C}^{(3)}=\frac{1+i \sqrt{3}}{2}, & \mathcal{A}(S(3,3,3))=\frac{\sqrt{3}}{2} .
\end{array}
$$

The situation for $S(2,2,2,2)$ is slightly different, since there is flexibility besides rescaling. For $s, t \in \mathbb{R}$ with $s>0$ we consider in $\mathbb{E}$ the quadrilateral $Q_{s, t}$ with corners

$$
\widetilde{A}^{(2)}=0, \quad \widetilde{B}^{(2)}=\frac{1}{s}+i t, \quad \widetilde{C}^{(2)}=\frac{1}{s}+i(s+t), \quad \widetilde{D}^{(2)}=i s
$$

and we define $\Gamma_{(2,2,2,2)}^{s, t}$ as the group generated by the rotations of angle $\pi$ around these points. Then the action of $\Gamma_{(2,2,2,2)}^{s, t}$ on $\mathbb{E}$ defines on $S(2,2,2,2)$ a Euclidean structure of area 2, and a fundamental domain is given by the union of $Q_{s, t}$ with any translate of itself having an edge in common with itself, as shown in Fig. 6-left. When $S(2,2,2,2)$ plays the rôle of $X$ in $\widetilde{X} \rightarrow X$ we will endow it with the structure given by $s=1, t=0$, as shown in Fig. 6-right. It is an easy exercise to check that any other structure with area 2 is defined by $\Gamma_{(2,2,2,2)}^{s, t}$ for some $s, t$.

General geometric tools. Our analysis of the candidate covers (1)-(7) of Fig. 4 relies on certain facts that we will use repeatedly. The first is the exact determination of the lifts of the cone points, that we now describe. For any of our four Euclidean $X$ 's, with the structure $\pi: \mathbb{E} \rightarrow X=\mathbb{E} / \Gamma$ we have fixed, and any vertex $\widetilde{V}^{(p)}$ of the fundamental domain for $\Gamma$ described above, we set $V^{(p)}=\pi\left(\widetilde{V}^{(p)}\right)$, so that its cone order is $p$. Then $\pi^{-1}\left(V^{(p)}\right)$ will be some set $\left\{\widetilde{V}_{j}^{(p)}\right\} \subset \mathbb{E}$, with $j$ varying in a suitable set of indices. The exact lists of lifts are as follows:

$$
\begin{array}{ll}
S(2,4,4): & \text { (see Fig. 77left), } \\
\widetilde{A}_{a, b}^{(2)}=a+i b & a, b \in \mathbb{Z}, a \neq b(\bmod 2), \\
\widetilde{B}_{a, b}^{(4)}=a+i b & a, b \in \mathbb{Z}, a \equiv b \equiv 0(\bmod 2), \\
\widetilde{C}_{a, b}^{(4)}=a+i b & a, b \in \mathbb{Z}, a \equiv b \equiv 1(\bmod 2) ;
\end{array}
$$



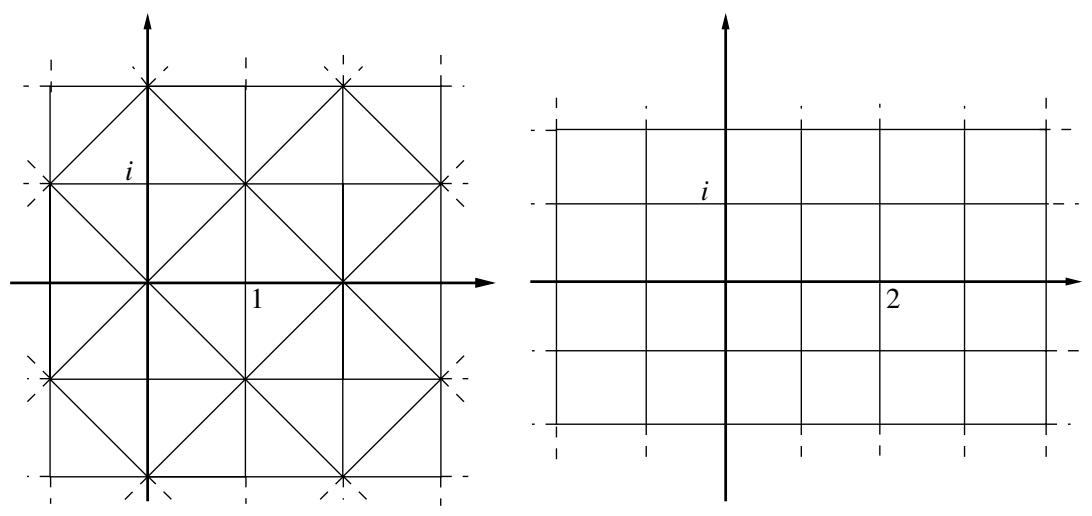

Figure 7 . Tessellations of $\mathbb{E}$ induced by the geometric structures fixed on $S(2,4,4)$ and $S(2,2,2,2)$
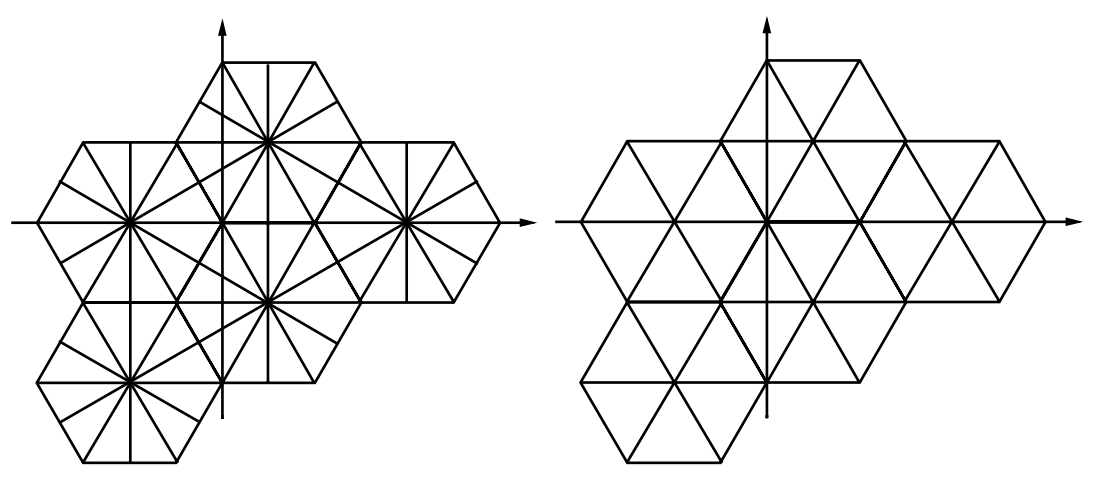

Figure 8 . Tessellations of $\mathbb{E}$ induced by the geometric structures fixed on $S(2,3,6)$ and $S(3,3,3)$

$S(2,2,2,2): \quad$ (see Fig. 7-right),

$$
\begin{array}{ll}
\widetilde{A}_{a, b}^{(2)}=a+i b & a, b \in \mathbb{Z}, a \equiv b \equiv 0(\bmod 2), \\
\widetilde{B}_{a, b}^{(2)}=a+i b & a, b \in \mathbb{Z}, a \equiv 1, b \equiv 0(\bmod 2), \\
\widetilde{C}_{a, b}^{(2)}=a+i b & a, b \in \mathbb{Z}, a \equiv b \equiv 1(\bmod 2), \\
\widetilde{D}_{a, b}^{(2)}=a+i b & a, b \in \mathbb{Z}, a \equiv 0, b \equiv 1(\bmod 2) ; \\
S(2,3,6): & \text { with } \omega=\frac{1+i \sqrt{3}}{2}(\text { see Fig. 8-left }), \\
\widetilde{A}_{a, b}^{(2)}=\frac{1}{2}(a+\omega b) & a, b \in \mathbb{Z} \text { not both even, } a-b \equiv 1(\bmod 3), \\
\widetilde{B}_{a, b}^{(3)}=a+\omega b & a, b \in \mathbb{Z}, a-b \neq \equiv(\bmod 3), \\
\widetilde{C}_{a, b}^{(6)}=a+\omega b & a, b \in \mathbb{Z}, a-b \equiv 2(\bmod 3) ;
\end{array}
$$

$S(3,3,3): \quad \quad$ with $\omega=\frac{1+i \sqrt{3}}{2}$ (see Fig. 8 8 right),

$$
\begin{array}{ll}
\widetilde{A}_{a, b}^{(3)}=a+\omega b, & a, b \in \mathbb{Z}, a-b \equiv 0(\bmod 3), \\
\widetilde{B}_{a, b}^{(3)}=a+\omega b, & a, b \in \mathbb{Z}, a-b \equiv 1(\bmod 3), \\
\widetilde{C}_{a, b}^{(3)}=a+\omega b, & a, b \in \mathbb{Z}, a-b \equiv 2(\bmod 3) .
\end{array}
$$


Also important for us will be the symmetries of our Euclidean $X$ 's. More precisely we will use the following facts:

- There exists a symmetry of $S(2,4,4)$ switching $B^{(4)}$ and $C^{(4)}$.

- Any permutation of $A^{(3)}, B^{(3)}, C^{(3)}$ is induced by a symmetry of $S(3,3,3)$.

- Any permutation of $A^{(2)}, B^{(2)}, C^{(2)}, D^{(2)}$ is induced by a symmetry of $S(2,2,2,2)$.

Here is another tool we will use very often. As one sees, if $\Gamma<\operatorname{Isom}^{+}(\mathbb{E})$ defines a Euclidean structure on a 2-orbifold $X=\mathbb{E} / \Gamma$, then $\Gamma$ has a maximal torsion-free subgroup, a rank-2 lattice $\Lambda(\Gamma)$. For $u \in \mathbb{C}$, let $\tau_{u}$ denote the translation $z \mapsto z+u$. Then the lattices for the groups we have fixed are as follows:

$$
\begin{aligned}
& \Lambda_{(2,4,4)}=\left\langle\tau_{2}, \tau_{2 i}\right\rangle, \quad \Lambda_{(2,2,2,2)}^{s, t}=\left\langle\tau_{2 i s}, \tau_{2\left(\frac{1}{s}+i t\right)}\right\rangle, \\
& \Lambda_{(2,3,6)}=\Lambda_{(3,3,3)}=\left\langle\tau_{i \sqrt{3}}, \frac{\tau_{3+i \sqrt{3}}}{2}\right\rangle .
\end{aligned}
$$

Moreover the following holds:

Lemma 3.2. Let $\widetilde{\Gamma}, \Gamma<\operatorname{Isom}^{+}(\mathbb{E})$ define Euclidean orbifolds $\widetilde{X}=\mathbb{E} / \widetilde{\Gamma}$ and $X=$ $\mathbb{E} / \Gamma$. Suppose that $\Lambda(\widetilde{\Gamma})=\left\langle\tau_{\widetilde{u}_{1}}, \tau_{\widetilde{u}_{2}}\right\rangle$ and $\Lambda(\Gamma)=\left\langle\tau_{u_{1}}, \tau_{u_{2}}\right\rangle$. Let $\widetilde{f}: \mathbb{E} \rightarrow \mathbb{E}$ given by $\widetilde{f}(z)=\lambda \cdot z+\mu$ have an associated orbifold cover $\widetilde{X} \rightarrow X$. Then $\lambda \cdot \widetilde{u}_{1}$ and $\lambda \cdot \widetilde{u}_{2}$ are integer linear combinations of $u_{1}$ and $u_{2}$.

Proof. The map $\tilde{f}$ induces a homomorphism $\tilde{f}_{*}: \widetilde{\Gamma} \rightarrow \Gamma$ given by $\widetilde{f}_{*}\left(\tau_{u}\right)=\tau_{\lambda \cdot u}$ which maps $\Lambda(\widetilde{\Gamma})$ to $\Lambda(\Gamma)$, and the conclusion easily follows.

Exceptions and geometric realizations. We will now state and prove 8 theorems corresponding to the cases (0)-(7) of Fig. 4, thus establishing Theorem 0.8 . Cases (1)-(3) imply in particular Theorems 0.4 0.5, and 0.6 .

Theorem 3.3 (Case (0) in Fig. 4). The candidate surface branched covers having associated candidate $T \rightarrow X$ are

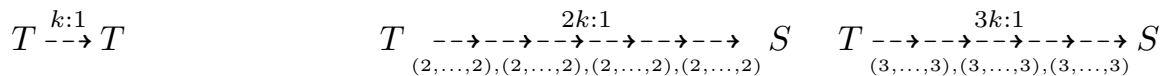

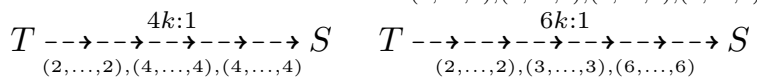

with arbitrary $k \geqslant 1$, and they are all realizable.

Proof. The first assertion and realizability of any $T \stackrel{k: 1}{\rightarrow} T$ are easy. For any $X \neq T$, let $X=\mathbb{E} / \Gamma$ and identify $T$ to $\mathbb{E} / \Lambda(\Gamma)$. Since $\Lambda(\Gamma)<\Gamma$ we have an associated orbifold cover $T \rightarrow X$, which realizes the relevant $T \rightarrow X$ in the special case $k=1$. The conclusion follows by taking compositions.

Theorem 3.4 (Case (1) in Fig. (4). The candidate surface branched covers having associated candidate $S(2,4,4) \stackrel{d: 1}{\rightarrow} S(2,4,4)$ are

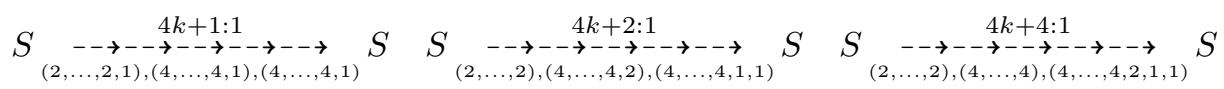

for $k \geqslant 1$, and they are realizable if and only if, respectively:

- $d=x^{2}+y^{2}$ for some $x, y \in \mathbb{N}$ of different parity;

- $d=2\left(x^{2}+y^{2}\right)$ for some $x, y \in \mathbb{N}$ of different parity;

- $d=4\left(x^{2}+y^{2}\right)$ for some $x, y \in \mathbb{N}$ not both zero. 
Proof. A candidate $S(2,4,4) \stackrel{d: 1}{\rightarrow-\rightarrow} S(2,4,4)$ can be complemented with any one of the following covering instructions:

$$
\begin{array}{ll}
2 \rightarrow 2,4 \rightarrow 4,4 \rightarrow 4, & 2 \rightarrow 4,(4,4) \rightarrow 4, \quad(2,4,4) \rightarrow 4, \\
2 \rightarrow 2,(4,4) \rightarrow 4, & (2,4) \rightarrow 4,4 \rightarrow 4
\end{array}
$$

and it is very easy to see that the first three come from the candidate surface branched covers of the statement, while the last two do not come from any candidate cover (recall that the simplified version $\ell(\Pi)=d+2$ (2) of the Riemann-Hurwitz formula must be satisfied).

Now suppose there is a realization $f: S(2,4,4) \stackrel{d: 1}{\longrightarrow} S(2,4,4)$ of one of the three relevant candidate covers. Proposition[1.3]implies that there is a geometric universal cover $\widetilde{\pi}: \mathbb{E} \rightarrow S(2,4,4)$ and an affine map $\widetilde{f}: \mathbb{E} \rightarrow \mathbb{E}$ with $\pi \circ \widetilde{f}=f \circ \widetilde{\pi}$. But the Euclidean structure of $S(2,4,4)$ is unique up to scaling, so $\widetilde{\pi}=\pi$. If $\widetilde{f}(z)=\lambda \cdot z+\mu$, since $\Lambda_{(2,4,4)}=\left\langle\tau_{2}, \tau_{2 i}\right\rangle$, Lemma 3.2 implies that $\lambda=n+i m$ for some $n, m \in \mathbb{Z}$, whence $d=n^{2}+m^{2}$.

We now employ the notation of (77) and note that for all three candidates we can assume, by the symmetry of $S(2,4,4)$, that $f\left(B^{(4)}\right)=B^{(4)}$, whence that $\widetilde{f}\left(\widetilde{B}_{0,0}^{(4)}\right)=\widetilde{B}_{0,0}^{(4)}$, namely $\mu=0$. We then proceed separately for the three candidates. For the first one we have $f\left(A^{(2)}\right)=A^{(2)}$, so $\widetilde{f}\left(\widetilde{A}_{1,0}^{(2)}\right)=\lambda$ is some $\widetilde{A}_{*}^{(2)}$. Therefore $n$ and $m$ have different parity, and we can set $x=|n|$ and $y=|m|$ getting that $d=x^{2}+y^{2}$ for $x, y \in \mathbb{N}$ of different parity. Conversely if $d$ has this form we define $\widetilde{f}(z)=(x+i y) \cdot z$. Then

$\widetilde{f}\left(\widetilde{A}_{1,0}^{(2)}\right)=\widetilde{A}_{x, y}^{(2)}, \quad \widetilde{f}\left(\widetilde{B}_{0,0}^{(4)}\right)=\widetilde{B}_{0,0}^{(4)}, \quad \widetilde{f}\left(\widetilde{C}_{1,1}^{(4)}\right)=(x-y)+i(x+y)=\widetilde{C}_{x-y, x+y}^{(4)}$,

where the last equality depends on the fact that $x-y \equiv x+y \equiv 1(\bmod 2)$. It easily follows that $\tilde{f}$ induces a realization of the candidate.

For the second candidate, $f\left(A^{(2)}\right)=C^{(4)}$; hence $\widetilde{f}\left(\widetilde{A}_{1,0}^{(2)}\right)=\lambda$ is some $\widetilde{C}_{*}^{(4)}$, namely $n$ and $m$ are odd. Setting $x=\frac{1}{2}|n+m|$ and $y=\frac{1}{2}|n-m|$ we see that $x, y \in \mathbb{N}$ have different parity and $d=2\left(x^{2}+y^{2}\right)$. Conversely if $d=2\left(x^{2}+y^{2}\right)$ with $x, y$ of different parity, we define $\widetilde{f}(z)=((x+y)+i(x-y)) \cdot z$. Then

$$
\widetilde{f}\left(\widetilde{A}_{1,0}^{(2)}\right)=\widetilde{C}_{x+y, x-y}^{(4)}, \quad \tilde{f}\left(\widetilde{B}_{0,0}^{(4)}\right)=\widetilde{B}_{0,0}^{(4)}, \quad \tilde{f}\left(\widetilde{C}_{1,1}^{(4)}\right)=\widetilde{B}_{2 y, 2 x}^{(4)},
$$

from which it is easy to see that $\tilde{f}$ induces a realization of the candidate.

For the last candidate, $f\left(A^{(2)}\right)=B^{(4)}$; hence $\tilde{f}\left(\widetilde{A}_{1,0}^{(2)}\right)$ is some $\widetilde{B}_{*}^{(4)}$, so $n$ and $m$ are even. Setting $x=\frac{1}{2}|n|$ and $y=\frac{1}{2}|m|$ we see that $d=4\left(x^{2}+y^{2}\right)$ for $x, y \in \mathbb{N}$. Conversely if $d=4\left(x^{2}+y^{2}\right)$ and we define $\widetilde{f}(z)=(2 x+2 i y) \cdot z$, then

$$
\widetilde{f}\left(\widetilde{A}_{1,0}^{(2)}\right)=\widetilde{B}_{2 x, 2 y}^{(4)}, \quad \widetilde{f}\left(\widetilde{B}_{0,0}^{(4)}\right)=\widetilde{B}_{0,0}^{(4)}, \quad \widetilde{f}\left(\widetilde{C}_{1,1}^{(4)}\right)=\widetilde{B}_{2(x-y), 2(x+y)}^{(4)} ;
$$

therefore $\tilde{f}$ induces a realization of the candidate.

Remark 3.5. One feature of the above proof is worth pointing out. After assuming that some degree- $d$ candidate $S(2,4,4) \rightarrow S(2,4,4)$ is realized by some map, we have always used only "two-thirds" of the branching instruction to show that $d$ has the appropriate form. The same phenomenon will occur in all the following proofs, except that of Theorem 3.9, Note however that to check that some $\tilde{f}$ defined starting 

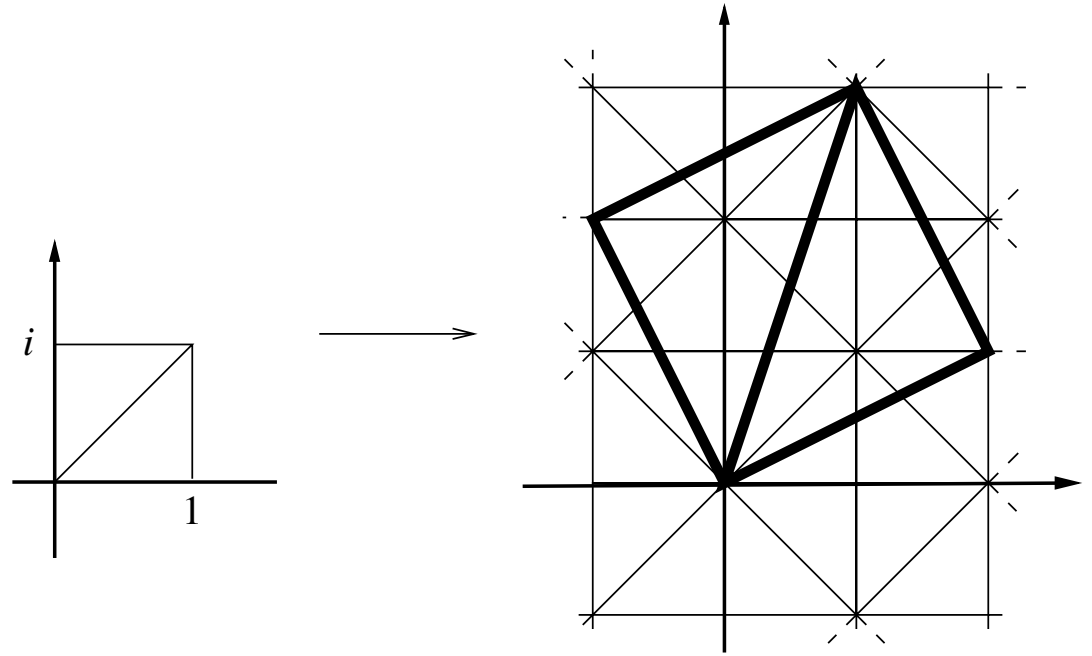

Figure 9. A map $\tilde{f}: \mathbb{E} \rightarrow \mathbb{E}$ inducing a degree-5 cover of $S(2,4,4)$ onto itself

from a degree $d$ with appropriate form induces a realization of the corresponding candidate, we had to check all three conditions.

As an illustration of the previous proof, we provide in Fig. 9 a description of the map $\tilde{f}: \mathbb{E} \rightarrow \mathbb{E}$ inducing a realization of $S \underset{\substack{-\rightarrow-\rightarrow-1 \rightarrow-\rightarrow \\(2,2,1),(4,1),(4,1)}}{\ln }$.

Theorem 3.6 (Case (2) in Fig. 4). The candidate surface branched covers having associated candidate $S(2,3,6) \stackrel{d: 1}{\rightarrow} S(2,3,6)$ are

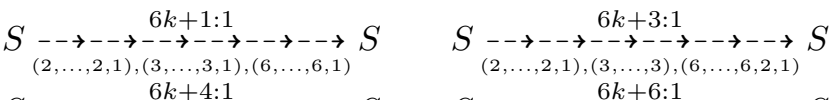

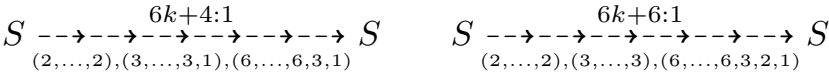

with $k \geqslant 1$, and they are realizable if and only if, respectively:

- $d=x^{2}+x y+y^{2}$ with $x, y \in \mathbb{N}$ not both even and $x \not \equiv y(\bmod 3)$;

- $d=3\left(x^{2}+3 x y+3 y^{2}\right)$ with $x, y \in \mathbb{N}$ not both even;

- $d=12\left(x^{2}+3 x y+3 y^{2}\right)+16$ with $x, y \in \mathbb{N}$;

- $d=12\left(x^{2}+3 x y+3 y^{2}\right)$ with $x, y \in \mathbb{N}$.

Proof. A candidate orbifold cover $S(2,3,6) \rightarrow S(2,3,6)$ can be complemented with the covering instructions

$$
\begin{array}{ll}
2-\rightarrow 2,3-\rightarrow 3,6 \rightarrow 6 & 2-\rightarrow 2,(3,6) \rightarrow 6, \\
3-\rightarrow 3,(2,6)-\rightarrow 6, & (2,3,6)-\rightarrow 6,
\end{array}
$$

which are associated to the candidate surface branched covers of the statement (formula (2) is always satisfied in this case).

The scheme of the proof is now as for case (1): we consider the universal cover $\pi: \mathbb{E} \rightarrow S(2,3,6)$ we have fixed, we assume that a map $f: S(2,3,6) \stackrel{d: 1}{\longrightarrow} S(2,3,6)$ realizing some candidate cover exists, we use Proposition 1.3 to find $\tilde{f}: \mathbb{E} \rightarrow \mathbb{E}$ with $\widetilde{f}(z)=\lambda \cdot z+\mu$ and $\pi \circ \widetilde{f}=f \circ \pi$, and we show that $d=|\lambda|^{2}$ has the appropriate 
form. Moreover essentially the same calculations will also allow us to prove the converse. We will always use the notation fixed in (9), in particular $\omega=\frac{1+i \sqrt{3}}{2}$.

We first apply Lemma 3.2 . Since $\Lambda_{(2,3,6)}=\left\langle\tau_{i \sqrt{3}}, \tau_{\frac{3+i \sqrt{3}}{2}}\right\rangle$ we must have

$$
\lambda \cdot i \sqrt{3}=n \cdot i \sqrt{3}+m \cdot \frac{3+i \sqrt{3}}{2}
$$

for some $n, m \in \mathbb{Z}$, which easily implies that

$$
\lambda=n+\frac{m}{2}-i \sqrt{3} \frac{m}{2}=(n+m)-\omega m
$$

therefore $d=n^{2}+n m+m^{2}$. Notice that Lemma 3.2 does not give any other condition, because $\lambda \cdot \frac{3+i \sqrt{3}}{2}=(-m) \cdot i \sqrt{3}+(n+m) \cdot \frac{3+i \sqrt{3}}{2}$.

We now analyze our four candidates, starting from the first one. Since

$$
f\left(A^{(2)}\right)=A^{(2)}, \quad f\left(B^{(3)}\right)=B^{(3)}, \quad f\left(C^{(6)}\right)=C^{(6)}
$$

we can assume $\tilde{f}\left(\widetilde{B}_{0,0}^{(3)}\right)=\widetilde{B}_{0,0}^{(3)}$, so $\mu=0$, and $\widetilde{f}\left(\widetilde{A}_{1,0}^{(2)}\right)=\widetilde{A}_{a, b}^{(2)}$ for some $a, b \in \mathbb{Z}$ not both even with $a-b \equiv 1(\bmod 3)$. Since $a=n+m$ and $b=-m$ we deduce that $n, m$ are not both even and $n-m \equiv 1(\bmod 3)$. If $n, m \geqslant 0$ or $n, m \leqslant 0$ we set $x=|n|$ and $y=|m|$, getting $d=x^{2}+x y+y^{2}$ with $x, y \in \mathbb{N}$ not both even and $x \not \equiv y(\bmod 3)$. Otherwise we have $n>0>m$ up to permutations. If $n \geqslant-m$ we set $x=n+m$ and $y=-m$; otherwise $x=n$ and $y=-n-m$, and again we have $d=x^{2}+x y+y^{2}$ with $x, y \in \mathbb{N}$ not both even and $x \not \equiv y(\bmod 3)$.

Conversely, assume $d=x^{2}+x y+y^{2}$ with $x, y \in \mathbb{N}$ not both even and $x \not \equiv y$ (mod 3$)$. Up to changing sign to both $x$ and $y$ we can suppose that $x-y \equiv 1$ $(\bmod 3)$ and define $\tilde{f}(z)=((x+y)-\omega y) \cdot z$. Of course $\widetilde{f}\left(\widetilde{B}_{0,0}^{(3)}\right)=\widetilde{B}_{0,0}^{(3)}$, the above calculations show that $\tilde{f}\left(\widetilde{A}_{1,0}^{(2)}\right)=\widetilde{A}_{x+y,-y}^{(2)}$, and using the identity $\omega^{2}=\omega-1$ we have $\tilde{f}\left(\widetilde{C}_{0,1}^{(6)}\right)=(x+y-\omega y) \cdot \omega=y+\omega x=\widetilde{C}_{y, x}^{(6)}$, where the last equality depends on the fact that $y-x \equiv-(x-y) \equiv 2(\bmod 3)$. This easily implies that $\tilde{f}$ induces a realization of the first candidate.

Let us turn to the second candidate. Since

$$
f\left(A^{(2)}\right)=A^{(2)}, \quad f\left(B^{(3)}\right)=C^{(6)}, \quad f\left(C^{(6)}\right)=C^{(6)},
$$

we can assume $\tilde{f}\left(\widetilde{B}_{0,0}^{(3)}\right)=\widetilde{C}_{0,1}^{(6)}$, namely $\mu=\omega$. In addition $\tilde{f}\left(\widetilde{A}_{1,0}^{(2)}\right)=\widetilde{A}_{a, b}^{(2)}$ for some $a, b \in \mathbb{Z}$ not both even with $a-b \equiv 1(\bmod 3)$. Since $a=n+m$ and $b=2-m$ we deduce that $n, m$ are not both even and $n \equiv m(\bmod 3)$. Setting $x=n$ and $y=(m-n) / 3$ we then get $d=3\left(x^{2}+3 x y+3 y^{2}\right)$ for $x, y \in \mathbb{Z}$ not both even. Reducing to the case $x, y \in \mathbb{N}$ is a routine matter that we leave to the reader.

Conversely, assume $d=3\left(x^{2}+3 x y+3 y^{2}\right)$ for $x, y \in \mathbb{N}$ not both even, set $n=x$ and $m=x+3 y$ and define

$$
\widetilde{f}(z)=((n+m)-\omega m) \cdot z+\omega .
$$

Then $\tilde{f}\left(\widetilde{A}_{1,0}^{(2)}\right)$ is some $\widetilde{A}_{*}^{(2)}$ by the above calculations, while

$$
\widetilde{f}\left(\widetilde{C}_{0,1}^{(6)}\right)=\widetilde{f}(\omega)=(x+3 y)+\omega(x+1),
$$


which is some $\widetilde{C}_{*}^{(6)}$ because $(x+3 y)-(x+1) \equiv 2(\bmod 3)$. It follows that $\tilde{f}$ induces a realization of the candidate.

For the third candidate we have

$$
f\left(A^{(2)}\right)=C^{(6)}, \quad f\left(B^{(3)}\right)=B^{(3)}, \quad f\left(C^{(6)}\right)=C^{(6)}
$$

so we can take $\mu=0$ and $\tilde{f}\left(\widetilde{A}_{1,0}^{(2)}\right)=\widetilde{C}_{a, b}^{(6)}$ for some $a, b \in \mathbb{Z}$ with $a-b \equiv$ $2(\bmod 3)$. Since $a=(n+m) / 2$ and $b=-m / 2$ we have that $n$ and $m$ are even and $n-m \equiv 1(\bmod 3)$. So we can define $x=\frac{n+4}{2}$ and $y=\frac{m-n-8}{6}$ and we have $d=12\left(x^{2}+3 x y+3 y^{2}\right)+16$ with $x, y \in \mathbb{Z}$. Again it is easy to reduce to the case $x, y \in \mathbb{N}$.

Conversely, suppose $d=12\left(x^{2}+3 x y+3 y^{2}\right)+16$ with $x, y \in \mathbb{N}$, set $n=2(x-2)$ and $m=2(x+3 y+2)$, and define $\widetilde{f}(z)=((n+m)-\omega m) \cdot z$. Then of course $\widetilde{f}\left(\widetilde{B}_{0,0}^{(3)}\right)=\widetilde{B}_{0,0}^{(3)}$, the above calculations show that $\widetilde{f}\left(\widetilde{A}_{1,0}^{(2)}\right)$ is some $\widetilde{C}_{*}^{(6)}$, while

$$
\widetilde{f}\left(\widetilde{C}_{0,1}^{(6)}\right)=\widetilde{f}(\omega)=2(x+3 y+2)+\omega 2(x-2),
$$

which is some $\widetilde{C}_{*}^{(6)}$ because $2(x+3 y+2)-2(x-2) \equiv 2(\bmod 3)$; hence $\tilde{f}$ induces a realization of the candidate.

For the last candidate,

$$
f\left(A^{(2)}\right)=C^{(6)}, \quad f\left(B^{(3)}\right)=C^{(6)}, \quad f\left(C^{(6)}\right)=C^{(6)},
$$

so we can assume $\tilde{f}\left(\widetilde{B}_{0,0}^{(3)}\right)=\widetilde{C}_{0,1}^{(6)}$, namely $\mu=\omega$. In addition $\tilde{f}\left(\widetilde{A}_{1,0}^{(2)}\right)=\widetilde{C}_{a, b}^{(6)}$ for some $a, b \in \mathbb{Z}$ with $a-b \equiv 2(\bmod 3)$. Since $a=(n+m) / 2$ and $b=(2-m) / 2$ we deduce that $n, m$ are both even and $n \equiv m(\bmod 3)$. Setting $x=n / 2$ and $y=(m-n) / 6$ we then get $d=12\left(x^{2}+3 x y+3 y^{2}\right)$ for $x, y \in \mathbb{Z}$, and again we can reduce to $x, y \in \mathbb{N}$.

Conversely, suppose $d=12\left(x^{2}+3 x y+3 y^{2}\right)$ for $x, y \in \mathbb{N}$, set $n=2 x$ and $m=2(x+3 y)$ and define $\tilde{f}(z)=((n+m)-\omega m) \cdot z+\omega$. Then $\tilde{f}\left(\widetilde{B}_{0,0}^{(3)}\right)=\widetilde{C}_{1,0}^{(6)}$ and $\tilde{f}\left(\widetilde{A}_{1,0}^{(2)}\right)$ is some $\widetilde{C}_{*}^{(6)}$ by the above calculations, while

$$
\widetilde{f}\left(\widetilde{C}_{0,1}^{(6)}\right)=\widetilde{f}(\omega)=(2 x+3 y)+(2 x+1) \omega,
$$

which is some $\widetilde{C}_{*}^{(6)}$ because $(2 x+3 y)-(2 x+1) \equiv 2(\bmod 3)$; hence $\tilde{f}$ induces a realization of the candidate.

Theorem 3.7 (Case (3) in Fig. 4). The candidate surface branched covers inducing $S(3,3,3) \stackrel{d: 1}{\stackrel{-}{\rightarrow}} S(3,3,3)$ are

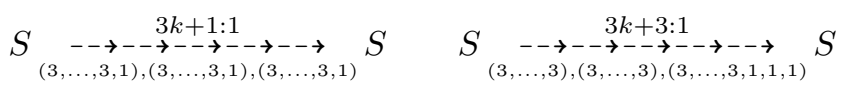

for $k \geqslant 1$, and they are realizable if and only if, respectively,

- $d=x^{2}+x y+y^{2}$ with $x, y \in \mathbb{N}$ and $x \neq \equiv y(\bmod 3)$;

- $d=3\left(x^{2}+3 x y+3 y^{2}\right)$ with $x, y \in \mathbb{N}$.

Proof. The possible covering instructions are

$$
3 \rightarrow 3,3-\rightarrow 3,3-\rightarrow 3, \quad(3,3)-\rightarrow 3,3-\rightarrow 3, \quad(3,3,3)-\rightarrow 3 .
$$


The second one is not associated to any candidate surface branched cover, and the other two are associated to the candidates in the statement.

We follow again the same scheme, using the notation of (10). If $\tilde{f}(z)=\lambda$. $z+\mu$ realizes a candidate, then, as in the previous proof, Lemma 3.2 implies that $\lambda=(n+m)-\omega m$ for $n, m \in \mathbb{Z}$, and $d=n^{2}+n m+m^{2}$. Moreover from the symmetry of $S(3,3,3)$ we can assume $f\left(A^{(3)}\right)=A^{(3)}$, whence $\widetilde{f}\left(\widetilde{A}_{0,0}^{(3)}\right)=\widetilde{A}_{0,0}^{(3)}$, namely $\mu=0$.

For the first candidate we have $f\left(B^{(3)}\right)=B^{(3)}$ up to symmetry, whence $\widetilde{f}\left(\widetilde{B}_{1,0}^{(3)}\right)=\lambda$ is some $\widetilde{B}_{a, b}^{(3)}$ with $a-b \equiv 1(\bmod 3)$; therefore $n-m \equiv 1(\bmod 3)$. Exactly as in the previous proof we deduce that $d=x^{2}+x y+y^{2}$ with $x, y \in \mathbb{N}$ and $x \not \equiv y(\bmod 3)$. The converse is proved as above. Switching signs if necessary we assume $x-y \equiv 1(\bmod 3)$, we set $\tilde{f}(z)=((x+y)-\omega y) \cdot z$ and note that $\tilde{f}\left(\widetilde{C}_{0,1}^{(3)}\right)=\tilde{f}(\omega)=y+\omega x$ is $\widetilde{C}_{y, x}^{(6)}$ because $y-x \equiv 2(\bmod 3)$.

For the second candidate, $f\left(B^{(3)}\right)=A^{(3)}$, whence $\widetilde{f}\left(\widetilde{B}^{(3)_{1,0}}\right)=\lambda$ is some $\widetilde{A}_{*}^{(3)}$, which implies that $n \equiv m(\bmod 3)$. Setting $x=n$ and $y=(m-n) / 3$ we see that $x, y \in \mathbb{Z}$ and $d=3\left(x^{2}+3 x y+y^{2}\right)$, and the conclusion is as usual.

Theorem 3.8 (Case (4) in Fig. 4). The candidate surface branched covers inducing some $S(2,2,2,2) \stackrel{d: 1}{-\rightarrow} S(2,2,2,2)$ are

$$
\begin{gathered}
2 k+1: 1 \\
S \begin{array}{c}
-\rightarrow--\rightarrow--\rightarrow--\rightarrow--\rightarrow--\rightarrow--\rightarrow \\
(2, \ldots, 2,1),(2, \ldots, 2,1),(2, \ldots, 2,1),(2, \ldots, 2,1)
\end{array}
\end{gathered} \quad S
$$

for $k \geqslant 1$. The first two are always realizable, the last one is if and only if $d$ is a multiple of 4.

Proof. The first assertion is easy (but note that now the Riemann-Hurwitz formula cannot be used in its simplified form (2); it reads $\ell(\Pi)=2 d+2$ ). The second assertion is proved as usual, except that we have to deal with the flexibility of $S(2,2,2,2)$. We assume that a map $f: S(2,2,2,2) \stackrel{d: 1}{\longrightarrow} S(2,2,2,2)$ realizing some candidate exists and we put on the target $S(2,2,2,2)$ the structure $\pi$ defined by $\Gamma_{(2,2,2,2)}^{1,0}$. Then we deduce from Lemma 1.3 that there exists a structure $\tilde{\pi}$ on the source $S(2,2,2,2)$ also with area 2 , and $\tilde{f}: \mathbb{E} \rightarrow \mathbb{E}$, such that $\tilde{f}(z)=\lambda \cdot z+\mu$ with $\pi \circ \tilde{f}=f \circ \tilde{\pi}$ and $d=|\lambda|^{2}$. Then $\tilde{\pi}$ is defined by some $\Gamma_{(2,2,2,2)}^{s, t}$.

We first note that by the symmetry of $S(2,2,2,2)$ we can assume $\mu=0$. Then we apply Lemma 3.2 . Since

$$
\Lambda_{(2,2,2,2)}^{1,0}=\left\langle\tau_{2 i}, \tau_{2}\right\rangle, \quad\left\langle\tau_{2 i s}, \tau_{2\left(\frac{1}{s}+i t\right)}\right\rangle
$$

there exist $m, n, p, q \in \mathbb{Z}$ such that

$$
\left\{\begin{array}{l}
\lambda \cdot i s=n+i m \\
\lambda \cdot\left(\frac{1}{s}+i t\right)=p+i q
\end{array}\right.
$$


and some easy computations show that all the relevant quantities can be determined explicitly in terms of $n, m, p, q$, namely:

$$
s=\sqrt{\frac{n^{2}+m^{2}}{p m-q n}}, \quad t=\frac{s p}{n}-\frac{m}{n s}, \quad \lambda=\frac{1}{s}(m-i n),
$$

so in particular $d=|\lambda|^{2}=p m-q n$. Note also that equations (11) already give us also the images of the lifts of the cone points.

For the first candidate we have $d=4 a \pm 1$ for some $a \geqslant 1$, we set $n=2 a, m=1$, $p= \pm 1, q=-2$, we compute $s, t, \lambda$ as above and we see that the corresponding map $\tilde{f}$ induces a realization of the candidate, because

$$
\begin{array}{ll}
\tilde{f}\left(\widetilde{A}^{(2)}\right)=\widetilde{A}_{0,0}^{(2)}, & \tilde{f}\left(\widetilde{B}^{(2)}\right)=\widetilde{B}_{ \pm 1,-2}^{(2)}, \\
\tilde{f}\left(\widetilde{C}^{(2)}\right)=\widetilde{C}_{2 a \pm 1,-1}^{(2)}, & \tilde{f}\left(\widetilde{D}^{(2)}\right)=\widetilde{D}_{2 a, 1}^{(2)} .
\end{array}
$$

For the second candidate we have $d=4 a+1 \pm 1$ for some $a \geqslant 1$, we set $n=2 a, m=1 \pm 1, p=1, q=-2$, we compute $s, t, \lambda$ as above and we see that the corresponding map $\tilde{f}$ induces a realization of the candidate, because

$$
\begin{array}{ll}
\tilde{f}\left(\widetilde{A}^{(2)}\right)=\widetilde{A}_{0,0}^{(2)}, & \tilde{f}\left(\widetilde{B}^{(2)}\right)=\widetilde{B}_{1,-2}^{(2)}, \\
\widetilde{f}\left(\widetilde{C}^{(2)}\right)=\widetilde{B}_{2 a+1, \pm 1-1}^{(2)}, & \widetilde{f}\left(\widetilde{D}^{(2)}\right)=\widetilde{A}_{2 a, 1 \pm 1}^{(2)} .
\end{array}
$$

For the last candidate each lift of a cone point has some $\widetilde{A}_{*}^{(2)}$ as its image; therefore $n, m, p, q$ must all be even, which implies that $d$ is a multiple of 4 , as prescribed in the statement. Conversely if $d=4 a$ for $a>1$ we set $n=m=q=2$, $p=2(a+1)$ we compute $s, t, \lambda$ as above and we see that the corresponding map $\tilde{f}$ induces a realization of the candidate, because

$$
\begin{array}{ll}
\tilde{f}\left(\widetilde{A}^{(2)}\right)=\widetilde{A}_{0,0}^{(2)}, & \tilde{f}\left(\widetilde{B}^{(2)}\right)=\widetilde{A}_{2(a+1), 2}^{(2)}, \\
\widetilde{f}\left(\widetilde{C}^{(2)}\right)=\widetilde{A}_{2(a+2), 4}^{(2)}, & \widetilde{f}\left(\widetilde{D}^{(2)}\right)=\widetilde{A}_{2,2}^{(2)} .
\end{array}
$$

The proof is complete.

Theorem 3.9 (Case (5) in Fig. 4). The candidate surface branched covers having associated candidate $S(3,3,3) \stackrel{d: 1}{\rightarrow} S(2,3,6)$ are

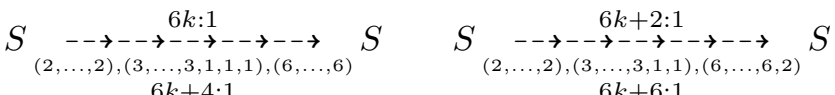

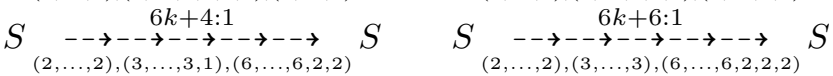

for $k \geqslant 1$, and they are realizable, respectively:

- if and only if $d=6\left(x^{2}+3 x y+3 y^{2}\right)$ for $x, y \in \mathbb{N}$;

- if and only if $d=2\left(x^{2}+x y+y^{2}\right)$ for $x, y \in \mathbb{N}$ and $x \neq \equiv(\bmod 3)$;

- never;

- if and only if $d=6\left(x^{2}+3 x y+3 y^{2}\right)$ for $x, y \in \mathbb{N}$.

Proof. The first assertion is easy. For the second one we proceed as above, except that now the Euclidean structure $\tilde{\pi}$ on $S(3,3,3)$ is not that we have fixed above, because its area should be $\frac{\sqrt{3}}{4}$ rather than $\frac{\sqrt{3}}{2}$, so the triangle $\Delta(3,3,3)$ must be 
rescaled by a factor $1 / \sqrt{2}$. The lattices to which we can apply Lemma 3.2 are therefore

$$
\frac{1}{\sqrt{2}} \cdot \Lambda_{(3,3,3)}=\left\langle\tau_{i \sqrt{\frac{3}{2}}}, \tau_{\frac{3+i \sqrt{3}}{2 \sqrt{2}}}\right\rangle, \quad \Lambda_{(2,3,6)}=\left\langle\tau_{i \sqrt{3}}, \frac{\tau_{\frac{3+i \sqrt{3}}{2}}}{}\right\rangle .
$$

As in the proof of Theorem 3.6 (except for the new factor) we deduce that

$$
\lambda=\sqrt{2} \cdot((n+m)-\omega m), \quad d=|\lambda|^{2}=2\left(n^{2}+n m+m^{2}\right) .
$$

Therefore $\tilde{f}$ maps the lifts of the cone points of $S(3,3,3)$ to

$$
\widetilde{f}(0)=\mu, \quad \tilde{f}\left(\frac{1}{\sqrt{2}}\right)=(n+m)-\omega m+\mu, \quad \widetilde{f}\left(\frac{\omega}{\sqrt{2}}\right)=m+n \omega+\mu .
$$

For the first candidate, all these points should be some $\widetilde{B}_{*}^{(3)}$ from (9), so we can assume $\mu=0$ and

$$
n+m-(-m) \not \equiv 2(\bmod 3), \quad m-n \not \equiv 2(\bmod 3) \quad \Rightarrow \quad n \equiv m(\bmod 3) .
$$

Setting $x=n$ and $y=(m-n) / 3$ we then see that $d=6\left(x^{2}+3 x y+3 y^{2}\right)$ for $x, y \in \mathbb{Z}$, and as above we can reduce to $x, y \in \mathbb{N}$, so $d$ has the appropriate form. The converse follows from the same computations: if $d=6\left(x^{2}+3 x y+3 y^{2}\right)$ we set $n=x$ and $m=x+3 y$ and we see that the corresponding $\widetilde{f}$ realizes the candidate.

For the second candidate, again $\mu=0$ and, by the symmetry of $S(3,3,3)$, we can assume $1 / \sqrt{2}$ is mapped to some $C_{*}^{(6)}$, namely $n-m \equiv 2(\bmod 3)$, so in particular $n \not \equiv m(\bmod 3)$. Therefore $d=2\left(x^{2}+x y+y^{2}\right)$ for some $x, y \in \mathbb{Z}$ with $x \not \equiv y(\bmod 3)$, and once again restricting to $x, y \in \mathbb{N}$ makes no difference, so $d$ has the prescribed form. The construction is easily reversible because if $n-m \equiv 2(\bmod 3)$, then $m-n \not \equiv 2(\bmod 3)$, which also proves that the third candidate is never realizable.

For the last candidate, we can assume $\mu=\omega$, and

$$
\begin{aligned}
& (n+m)-(1-m) \equiv 2(\bmod 3) \\
& m-(n+1) \equiv 2(\bmod 3)
\end{aligned} \quad \Rightarrow \quad n \equiv m(\bmod 3)
$$

and we conclude as for the first candidate.

Theorem 3.10 (Case (6) in Fig. (4). The candidate surface branched covers having associated candidate $S(2,2,2,2) \stackrel{d: 1}{\rightarrow} S(2,4,4)$ are

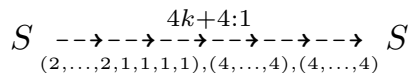

$$
\begin{aligned}
& S \rightarrow \begin{array}{c}
4 k+2: 1 \\
-\rightarrow--\rightarrow--\rightarrow--\rightarrow--\rightarrow--\rightarrow \quad S
\end{array}
\end{aligned}
$$

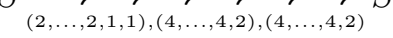

$$
\begin{aligned}
& S \begin{array}{c}
4 k+6: 1 \\
\underset{(2, \ldots, 2),(4, \ldots, 4,2,2,2),(4, \ldots, 4,2)}{--\rightarrow--\rightarrow--\rightarrow-1,4}
\end{array} S \\
& \begin{array}{c}
4 k+4: 1 \\
S \begin{array}{c}
--\rightarrow--\rightarrow--\rightarrow--\rightarrow--\rightarrow--\rightarrow \\
(2, \ldots, 2,1,1),(4, \ldots, 4,2,2),(4, \ldots, 4)
\end{array}
\end{array}
\end{aligned}
$$

for $k \geqslant 1$. The first four are always realizable, the fifth one is never, and the last one is if and only if $d$ is a multiple of 8.

Proof. Again we leave the first assertion to the reader and we proceed with the customary scheme. Since the area of the structure we have chosen on $S(2,4,4)$ is 1 , on $S(2,2,2,2)$ we will have a structure generated by the rotations of angle $\pi$ around points

$$
0, \quad \frac{1}{2 s}+i t, \quad \frac{1}{2 s}+i(s+t), \quad i s
$$


with $s, t \in \mathbb{R}$ and $s>0$. The lattices to which we must apply Lemma 3.2 are therefore $\left\langle\tau_{2 i s}, \tau_{\frac{1}{s}+2 i t}\right\rangle$ and $\Lambda_{(2,4,4)}=\left\langle\tau_{2}, \tau_{2 i}\right\rangle$, so

$$
\left\{\begin{array}{l}
\lambda \cdot 2 i s=2(n+i m) \\
\lambda \cdot\left(\frac{1}{s}+2 i t\right)=2(p+i q)
\end{array}\right.
$$

for some $n, m, p, q \in \mathbb{Z}$. Whence, after easy computations,

$$
s=\sqrt{\frac{n^{2}+m^{2}}{2(p m-q n)}}, \quad t=\frac{s p}{n}-\frac{m}{2 s n}, \quad \lambda=\frac{m-i n}{s} .
$$

In particular $d=2(p m-q n)$ and the images of the lifts of the cone points of $S(2,2,2,2)$ are

$$
\begin{array}{ll}
\widetilde{f}(0)=\mu, & \widetilde{f}\left(\frac{1}{2 s}+i t\right)=p+i q+\mu, \\
\widetilde{f}(i s)=n+i m+\mu, & \widetilde{f}\left(\frac{1}{2 s}+i(s+t)\right)=(p+n)+i(q+m)+\mu .
\end{array}
$$

The first four candidates are realized, respectively, with the following choices of $n, m, p, q, \mu$ :

\begin{tabular}{c|c|c|c|c}
$n$ & $m$ & $p$ & $q$ & $\mu$ \\
\hline$k+1$ & $k+1$ & 1 & -1 & 1 \\
$k$ & $k+1$ & 2 & 0 & 0 \\
$k$ & $k+1$ & 1 & -1 & 0 \\
$k$ & $k+1$ & 2 & 0 & 0
\end{tabular}

The fifth candidate is always exceptional because we can suppose $\mu=0$ and hence we should have that two of the pairs

$$
(p, q), \quad(n, m), \quad(p+n, q+m)
$$

consist of even numbers and the third one consists of odd numbers, which is impossible.

For the last candidate we have that $p, q, n, m$ must all be even, so $d=2(p m-q n)$ is a multiple of 8 . Conversely if $d=8 h$ we can realize the candidate with $n=q=0$, $m=2$ and $p=2 h$.

Theorem 3.11 (Case (7) in Fig. (4). The candidate surface branched covers having associated candidate $S(2,2,2,2) \stackrel{d: 1}{\rightarrow} S(2,3,6)$ are

$$
\begin{aligned}
& \begin{array}{c}
6 k: 1 \\
\begin{array}{c}
--\rightarrow--\rightarrow--\rightarrow--\rightarrow--\rightarrow--\rightarrow \\
(2, \ldots, 2,1,1,1,1),(3, \ldots, 3),(6, \ldots, 6)
\end{array}
\end{array} \\
& S \underset{(2, \ldots, 2,1,1),(3, \ldots, 3),(6, \ldots, 6,3,3)}{\substack{6 k+6: 1 \\
(--\rightarrow--\rightarrow--\rightarrow--\rightarrow--\rightarrow--\rightarrow}} S \\
& S \begin{array}{c}
6 k+12: 1 \\
\underset{(2, \ldots, 2),(3, \ldots, 3),(6, \ldots, 6,3,3,3,3)}{--\rightarrow--\rightarrow--\rightarrow--\rightarrow--\rightarrow--\rightarrow}
\end{array} S
\end{aligned}
$$

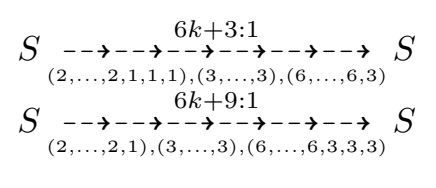

for $k \geqslant 1$. The first three are always realizable, the fourth one is never, and the last one is if and only if $d$ is a multiple of 12.

Proof. Once again we leave the first assertion to the reader and we follow the usual scheme. Since the area of $S(2,3,6)$ is $\sqrt{3} / 4$, on $S(2,2,2,2)$ we will have a structure generated by the rotations of angle $\pi$ around the points

$$
0, \quad \frac{\sqrt{3}}{8 s}+i t, \quad \frac{\sqrt{3}}{8 s}+i(s+t), \quad i s
$$


and we apply Lemma 3.2 to $\left\langle\tau_{2 i s}, \tau_{\frac{\sqrt{3}}{8 s}+2 i t}\right\rangle$ and $\Lambda_{(2,3,6)}=\left\langle\tau_{i \sqrt{3}}, \frac{\tau_{3+i \sqrt{3}}}{2}\right.$, so for some $n, m, p, q \in \mathbb{Z}$ we have

$$
\left\{\begin{array}{l}
\lambda \cdot 2 i s=n i \sqrt{3}+m \frac{3+i \sqrt{3}}{2}, \\
\lambda \cdot\left(\frac{\sqrt{3}}{4 s}+2 i t\right)=p i \sqrt{3}+q \frac{3+i \sqrt{3}}{2},
\end{array}\right.
$$

whence, after some calculations,

$$
\begin{aligned}
& s=\frac{1}{2} \sqrt{\frac{n^{2}+n m+m^{2}}{q n-p m}}, \quad t=\frac{q s}{m}-\frac{m+2 n}{8 m s}, \\
& \lambda=\frac{\sqrt{3}(m+2 n)-3 i m}{4 s}=\frac{\sqrt{3}}{2 s} \cdot((n+m)-m \omega),
\end{aligned}
$$

so in particular $d=|\lambda|^{2}=3(q n-p m)$. Moreover the following relations will readily allow us to determine the images under $\tilde{f}$ of the lifts of the cone points of $S(2,2,2,2)$ :

$$
\begin{aligned}
& \lambda \cdot i s=\frac{1}{2}((m-n)+(m+2 n) \omega), \\
& \lambda \cdot\left(\frac{\sqrt{3}}{8 s}+i t\right)=\frac{1}{2}((q-p)+(q+2 p) \omega) .
\end{aligned}
$$

For the first candidate we choose $\mu=\frac{1}{2}, p=q=2, n=k+1$ and $m=1$. The corresponding $\tilde{f}$ induces a realization because $d=6 k=3(q n-p m)$ and the images of the cone points are

$$
\begin{array}{ll}
\frac{1}{2}(1+0 \omega), & \frac{1}{2}((m-n+1)+(m+2 n) \omega), \\
\frac{1}{2}((q-p+1)+(q+2 p) \omega), & \frac{1}{2}((m+q-n-p+1)+(m+q+2 n+2 p) \omega),
\end{array}
$$

which are easily recognized to all have the form $\frac{1}{2}(a+b \omega)$ with $a, b$ not both even and $a-b \equiv 1(\bmod 3)$, so they equal to some $\widetilde{A}_{*}^{(2)}$.

For the second candidate we choose $\mu=\frac{1}{2}, n=2, m=1$ and

$$
q=k, p=-1 \quad \text { if } k \equiv 1(\bmod 2), \quad q=k+1, p=1 \quad \text { if } k \equiv 0(\bmod 2) .
$$

Then $d=6 k+3=3(q n-p m)$ and the images of the cone points are as before, but now the first three are some $\widetilde{A}_{*}^{(2)}$, while the last one has the form $a+b \omega$ with $a, b \in \mathbb{Z}$ and $a-b \equiv 2(\bmod 3)$, so it is some $\widetilde{C}_{*}^{(6)}$, so $\widetilde{f}$ induces a realization of the candidate.

For the third candidate we choose $\mu=\frac{1}{2}, m=q=2$ and

$$
n=k, p=-1 \quad \text { if } k \equiv 0(\bmod 2), \quad n=k+1, p=1 \quad \text { if } k \equiv 1(\bmod 2) .
$$

Then $d=6 k+6=3(q n-p m)$ and now the first two images are some $\widetilde{A}_{*}^{(2)}$ and the last two are some $\widetilde{C}_{*}^{(6)}$, so $\widetilde{f}$ induces a realization of the candidate.

For the fourth candidate we can once again suppose $\mu=\frac{1}{2}$. Since the images of the last three cone points must be some $\widetilde{C}_{*}^{(6)}$ we deduce that $m, q, m+q$ should be even and hence $n, p, n+p$ should be odd, which is impossible. 
Turning to the last candidate, we can suppose $\mu=\omega$. Then the images of the cone points are

$$
\begin{array}{ll}
1+0 \omega, & \frac{1}{2}((m-n)+(m+2 n+2) \omega), \\
\frac{1}{2}((q-p)+(q+2 p+2) \omega), & \frac{1}{2}((m+q-n-p)+(m+q+2 n+2 p+2) \omega)
\end{array}
$$

and they must all be some $\widetilde{C}_{*}^{(6)}$, so $n, m, p, q$ should all be even. Therefore $d=3(q n-p m)$ is a multiple of 12 . Conversely, if $d=12 h+12$, we realize the candidate with the choice $q=2 h, n=m=2$ and $p=-2$.

Congruences and density. We close this section with some elementary remarks about the numbers appearing in our main results, thus explaining in detail why Theorems 0.4 to 0.6 are implied by Theorems 3.4 to 3.7 . First of all we have:

$$
\begin{aligned}
& \left\{d \in \mathbb{N}: d=x^{2}+y^{2} \text { for } x, y \in \mathbb{N}, x \not \equiv y(\bmod 2)\right\} \\
& =\left\{d \in \mathbb{N}: d \equiv 1(\bmod 4), d=x^{2}+y^{2} \text { for } x, y \in \mathbb{N}\right\}, \\
& \left\{d \in \mathbb{N}: d=x^{2}+x y+y^{2} \text { for } x, y \in \mathbb{N} \text { not both even, } x \not \equiv y(\bmod 3)\right\} \\
& =\left\{d \in \mathbb{N}: d \equiv 1(\bmod 6), d=x^{2}+x y+y^{2} \text { for } x, y \in \mathbb{N}\right\}, \\
& \left\{d \in \mathbb{N}: d=x^{2}+x y+y^{2} \text { for } x, y \in \mathbb{N}, x \neq y(\bmod 3)\right\} \\
& =\left\{d \in \mathbb{N}: d \equiv 1(\bmod 3), d=x^{2}+x y+y^{2} \text { for } x, y \in \mathbb{N}\right\} .
\end{aligned}
$$

Moreover the statement made in the Introduction that in Theorems 0.4 to 0.6 the realizable degrees have zero asymptotic density means the following:

$$
\begin{aligned}
& \lim _{n \rightarrow \infty} \frac{1}{n} \cdot \#\left\{d \in \mathbb{N}: d \leqslant n, d=x^{2}+y^{2} \text { for } x, y \in \mathbb{N}\right\}=0 \\
& \lim _{n \rightarrow \infty} \frac{1}{n} \cdot \#\left\{d \in \mathbb{N}: d \leqslant n, d=x^{2}+x y+y^{2} \text { for } x, y \in \mathbb{N}\right\}=0 .
\end{aligned}
$$

\section{HyPERBOLIC TRIANGULAR ORBIFOLDS}

There is one crucial geometric fact underlying the proofs of our main results for the case of positive and zero Euler characteristic. Namely, in these cases the geometry (if any) of an orbifold with cone points is rigid (up to rescaling), with the single exception of $S(2,2,2,2)$, where the space of moduli is easy to compute anyway. Turning to the case of negative Euler characteristic, one knows that a hyperbolic 2-orbifold is rigid if and only if it is triangular, namely if it is based on the sphere and it has precisely three cone points. In this section we will show that only very few candidate surface branched covers have associated candidate covers between hyperbolic triangular 2-orbifolds:

Theorem 4.1. The candidate surface branched covers having associated candidate covers between triangular hyperbolic 2-orbifolds are precisely:
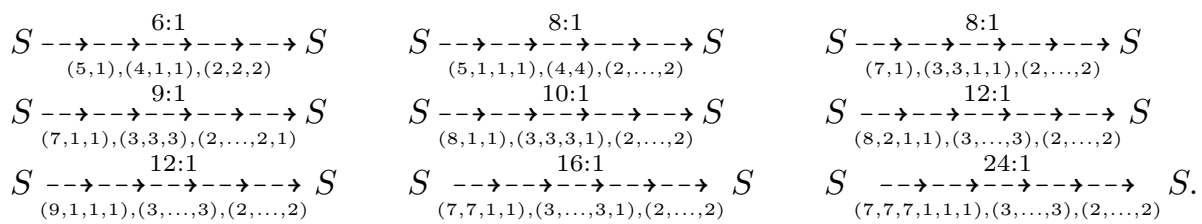

This result means that the geometric techniques employed above would require a substantial extension to be relevant for the hyperbolic case. Leaving this for the future, we will show here that for the candidate covers of Theorem 4.1 the geometric approach is not even necessary, since realizability can be fully analyzed 
TABLE 1 . The partitions $\Pi$ of 8 with $c(\Pi) \leqslant 3$

\begin{tabular}{c||c|c|c|c|c}
$\Pi$ & $(8)$ & $(6,1,1)$ & $(5,1,1,1)$ & $(4,2,2)$ & $(3,3,1,1)$ \\
\hline$\ell$ & 1 & 3 & 4 & 3 & 4 \\
\hline$c$ & 0 & 2 & 3 & 2 & 2 \\
\hline \hline$\Pi$ & $(7,1)$ & $(5,3)$ & $(4,4)$ & $(4,2,1,1)$ & $(2,2,2,2)$ \\
\hline$\ell$ & 2 & 2 & 2 & 4 & 4 \\
\hline$c$ & 1 & 2 & 0 & 3 & 0 \\
\hline \hline$\Pi$ & $(6,2)$ & $(5,2,1)$ & $(4,3,1)$ & $(3,3,2)$ & $(2,2,2,1,1)$ \\
\hline$\ell$ & 2 & 3 & 3 & 3 & 5 \\
\hline$c$ & 1 & 3 & 3 & 3 & 2
\end{tabular}

using a completely different technique, namely Grothendieck's dessins d'enfant [8, 24, already exploited in [18. We will show the following:

Proposition 4.2. Among the candidate covers of Theorem 4.1, the second and the eighth are exceptional and all other ones are realizable.

Let us now establish the results we have stated. The first proof requires the analysis of quite a few cases, some of which we will leave to the reader.

Proof of Theorem 4.1. Our argument is organized in three steps:

(I) analysis of the relevant surface candidate covers with degree $d \leqslant 11$;

(II) restrictions on the base of the associated candidate cover for $d \geqslant 12$;

(III) more restrictions on the cover and conclusion for $d \geqslant 12$.

SteP I. If $\Pi$ is a partition of an integer $d$, let us denote by $\ell(\Pi)$ its length (as above), and by $c(\Pi)$ the number of entries in $\Pi$ which are different from l.c.m.( $\Pi)$. To have an associated candidate cover between triangular 2-orbifolds (regardless of the geometry), a candidate surface branched cover of degree $d \geqslant 2$ must have the following properties:

- The number of branching points is 3 .

- If the partitions of $d$ are $\Pi_{1}, \Pi_{2}, \Pi_{3}$, then $c\left(\Pi_{1}\right)+c\left(\Pi_{2}\right)+c\left(\Pi_{3}\right)=3$.

To list all such candidate covers for a given $d$ then one has to:

- List all the partitions $\Pi$ of $d$ with $c(\Pi) \leqslant 3$.

- Find all possible triples $\left(\Pi_{1}, \Pi_{2}, \Pi_{3}\right)$ of partitions with $\ell\left(\Pi_{1}\right)+\ell\left(\Pi_{2}\right)+$ $\ell\left(\Pi_{3}\right)=d+2$ and $c\left(\Pi_{1}\right)+c\left(\Pi_{2}\right)+c\left(\Pi_{3}\right)=3$.

We have done this for $2 \leqslant d \leqslant 11$ and then we have singled out the candidate covers having associated hyperbolic 2-orbifold covers, getting the first five items of the statement. To illustrate how this works we will spell out here only the case $d=8$. The partitions $\Pi$ of 8 with $c(\Pi) \leqslant 3$ are those described in Table 1 with the corresponding values of $\ell$ and $c$.

The triples of such partitions such that $\ell$ and $c$ sum up to 10 and 3 respectively are shown in Table 2, together with the associated candidate orbifold cover and its geometric type. So we get the second and third item in the statement. 
TABLE 2. Triples of partitions of 8 having associated candidate covers between triangular orbifolds

\begin{tabular}{c|c|c|c|c}
\multicolumn{3}{c|}{$\Pi_{1}, \Pi_{2}, \Pi_{3}$} & Associated cover & Geometry \\
\hline \hline$(4,2,1,1)$ & $(4,4)$ & $(2,2,2,2)$ & $S(2,4,4) \rightarrow S(2,4,4)$ & $\mathbb{E}$ \\
\hline$(5,1,1,1)$ & $(4,4)$ & $(2,2,2,2)$ & $S(5,5,5) \rightarrow S(2,4,5)$ & $\mathbb{H}$ \\
\hline$(6,2)$ & $(3,3,1,1)$ & $(2,2,2,2)$ & $S(3,3,3) \rightarrow S(2,3,6)$ & $\mathbb{E}$ \\
\hline$(7,1)$ & $(3,3,1,1)$ & $(2,2,2,2)$ & $S(3,3,7) \rightarrow S(2,3,7)$ & $\mathbb{H}$
\end{tabular}

STEP II. Let us denote by $\widetilde{X}_{\stackrel{d: 1}{d} \rightarrow X}$ a candidate orbifold cover with $d \geqslant 12$ and hyperbolic $\widetilde{X}=S(\alpha, \beta, \gamma)$ and $X=S(p, q, r)$. Since

$$
0<-\chi^{\text {orb }}(\tilde{X})=1-\left(\frac{1}{\alpha}+\frac{1}{\beta}+\frac{1}{\gamma}\right)<1
$$

and $\chi^{\text {orb }}(\widetilde{X})=d \cdot \chi^{\text {orb }}(X)$, we deduce that

$$
0<-\chi^{\mathrm{orb}}(X)=1-\left(\frac{1}{p}+\frac{1}{q}+\frac{1}{r}\right)<\frac{1}{12} \Rightarrow \frac{11}{12}<\left(\frac{1}{p}+\frac{1}{q}+\frac{1}{r}\right)<1 .
$$

Assuming $p \leqslant q \leqslant r$ it is now very easy to check that the last inequality is satisfied only for $p=2, q=3,7 \leqslant r \leqslant 11$ and for $p=2, q=4, r=5$.

STEP III. If $\widetilde{X} \rightarrow X$ is a candidate 2 -orbifold cover with hyperbolic $\widetilde{X}=S(\alpha, \beta, \gamma)$ and $X=S(p, q, r)$, then the following must happen:

(a) Each of $\alpha, \beta, \gamma$ must be a divisor of some element of $\{p, q, r\}$.

(b) $\frac{\chi^{\text {orb }}(\widetilde{X})}{\chi^{\text {orb }}(X)}$ must be an integer $d$.

(c) There must exist three partitions of $d$ such that the associated candidate orbifold cover is $\widetilde{X} \rightarrow X$.

Successively imposing these conditions with each of the 5 orbifolds $X$ coming from Step II and restricting to $d \geqslant 12$ we have found the last four items in the statement. Again we only spell out one example here, leaving the other ones to the reader. Let $X$ be $S(2,3,8)$. Then the relevant hyperbolic $\widetilde{X}$ 's according to (a), excluding $X$ itself, are

$$
\begin{array}{lllll}
S(2,4,8) & S(3,3,4) & S(2,8,8) & S(3,3,8) & S(3,8,8) \\
S(3,4,4) & S(4,4,4) & S(4,4,8) & S(4,8,8) & S(8,8,8)
\end{array}
$$

and $d=\frac{\chi^{\mathrm{orb}}(\widetilde{X})}{\chi^{\mathrm{orb}}(X)}$ is always integer in this case, so point (b) is not an issue. However $d \leqslant 11$ in all cases but the last two (for instance, the case $\tilde{X}=S(3,8,8)$ corresponds to the fifth item in the statement). For $\widetilde{X}=S(4,8,8)$ we have $d=12$ and taking the partitions of 12 as the sixth item in the statement, we see that the associated orbifold cover is indeed $S(4,8,8) \rightarrow S(2,3,8)$. For $\widetilde{X}=S(8,8,8)$ we have $d=15$ and it is impossible to find partitions of 15 having the right associated candidate orbifold cover, because the cone point of order 2 in $X$, being covered by ordinary points of $\widetilde{X}$ only, should require a partition consisting of 2's only, which cannot exist because 15 is odd.

Carrying out the same analysis one gets the last two items in the statement for $X=S(2,3,7)$, the seventh item for $X=S(2,3,9)$, and nothing new for the other $X$ 's. This concludes Step III and the proof. 


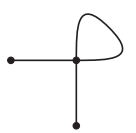

(1)

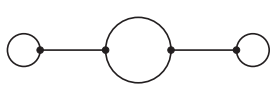

(6)

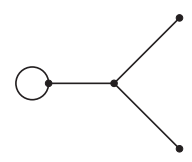

(3)

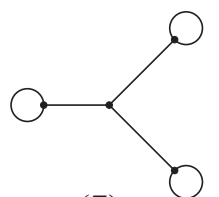

(7)

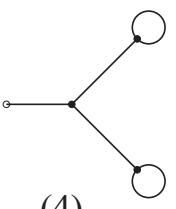

(4)

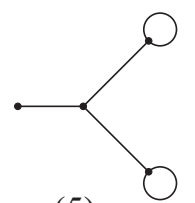

(5)

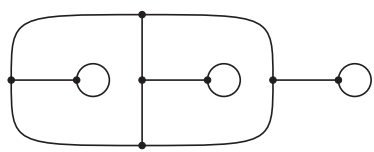

(9)

Figure 10. Dessins d'enfant for all candidate surfaced branched covers in Theorem 4.1 except the second and the eighth

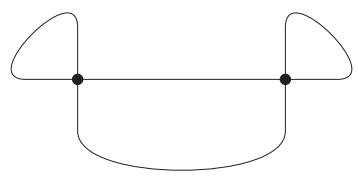

FIGURE 11. Exceptionality of $S \underset{\substack{-\rightarrow--\rightarrow-\rightarrow \rightarrow--\rightarrow--\rightarrow \\(5,1,1,1),(4,4),(2, \ldots, 2)}}{8: 1}$

As already mentioned, the next argument is based on a technique different from those used in the rest of this paper, namely Grothendieck's dessins d'enfant. We will not review this tool here, addressing the reader to [18].

Proof of Proposition 4.2. The dessins d'enfant proving the realizability of all candidate covers claimed to be realizable can be found in Fig. 10. The black vertices always correspond to the elements of the second partition in Theorem 4.1, and the white vertices to the entries of the third partition, while the regions correspond to the elements of the first partition. However 2-valent white vertices are never shown, except for the single 1-valent one in case (4).

The exceptionality of $S \underset{\substack{(5,1,1,1),(4,4),(2, \ldots, 2) \\: \rightarrow-\rightarrow-\rightarrow,}}{\longrightarrow} S$ is easy: a dessin relative to partitions $(4,4)$ and $(2,2,2,2)$ with at least two outer regions of length 1 must be as shown in Fig. 11] so the third partition is $(4,2,1,1)$, not $(5,1,1,1)$.

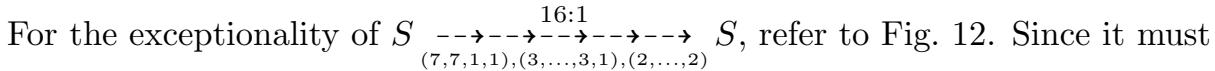
contain two length-1 regions, a dessin realizing it should be as in (a). The two marked germs of edges cannot be joined together or to the 1-valent vertex, so they go either to the same 3 -valent vertex as in (aa) or to different 3 -valent vertices as in (ab). Case (aa) is impossible because there is a region with 7 vertices, which will become more than 7 in the complete dessin. In case (ab) we examine where the marked germ of an edge could go, getting cases (aba) to (abd), always redrawn in a more convenient way. Cases (aba) and (abb) are impossible because of long regions. In cases (abc) and (abd) we examine where the marked edge could go in order not to create regions of length 5 or longer than 7 , and we see that in both cases there is only one possibility, namely (abca) and (abda). In both these cases, 


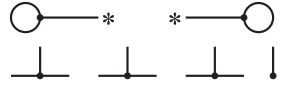

(a)

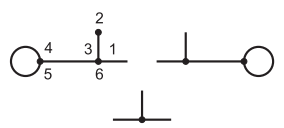

(aba)

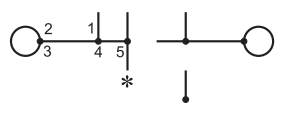

(abd)

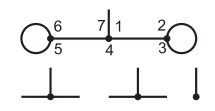

(a)

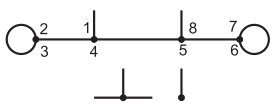

(abb)

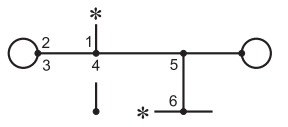

(abca)

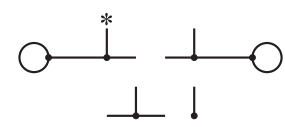

(ab)

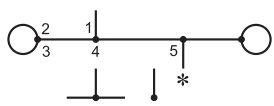

$(\mathrm{abc})$

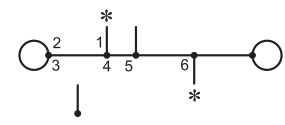

(abda)

FIgURE 12. Exceptionality of $\begin{gathered}\substack{-\rightarrow-\rightarrow-\rightarrow--\rightarrow-\rightarrow \rightarrow--\rightarrow--\rightarrow \\(7,7,1,1),(3, \ldots, 3,1),(2, \ldots, 2)} \\ S\end{gathered}$

because of the region of length already 6 , the two marked germs of edges should go to one and the same 3 -valent vertex, but there are no more available with two free germs of edges, so again we cannot complete the dessin in order to realize (8). Our argument is complete.

To double check our existence claims, we also provide permutations realizing the relevant covers, in the spirit explained at the end of Section 2

\begin{tabular}{l|lll} 
Case & Permutations \\
\hline \hline$(1)$ & $\sigma_{1}=(1,2,3,4,5)$ & $\sigma_{2}=(1,4,6,3)$ & $\sigma_{3}=(1,5)(2,3)(4,6)$ \\
\hline$(3)$ & $\sigma_{1}=(1,2,3,4,5,6,7)$ & $\sigma_{2}=(1,8,7)(2,6,4)$ & $\sigma_{3}=(1,8)(2,7)(3,4)(5,6)$ \\
\hline$(4)$ & $\sigma_{1}=(1,2,3,4,5,6,7)$ & $\sigma_{2}=(1,7,4)(2,3,8)(5,6,9)$ & $\sigma_{3}=(3,8)(2,4)(5,7)(6,9)$ \\
\hline$(5)$ & $\sigma_{1}=(1,2,3,4,5,6,7,8)$ & $\sigma_{2}=(1,7,4)(2,3,9)(5,6,10)$ & $\sigma_{3}=(1,8)(2,4)(3,9)(5,7)(6,10)$ \\
\hline$(6)$ & $\sigma_{1}=(1,2,3,4,5,6,7,8)(9,10)$ & $\sigma_{2}=(1,10,4)(2,3,12)(5,9,8)(6,7,11)$ \\
& $\sigma_{3}=(1,9)(2,4)(3,12)(5,10)(6,8)(7,11)$ \\
\hline$(7)$ & $\sigma_{1}=(1,2,3,4,5,6,7,8,9)$ & $\sigma_{2}=(1,2,10)(3,9,6)(4,5,11)(7,8,12)$ \\
\hline & $\sigma_{3}=(1,3)(2,10)(4,6)(5,11)(7,9)(8,12)$ \\
\hline & $\sigma_{1}=(1,2,3,4,5,6,7)(9,10,11,12,13,14,15)(17,18,19,20,21,22,23)$ \\
& $\sigma_{2}=(1,5,19)(2,18,10)(3,9,13)(4,12,20)(6,7,8)(11,17,21)(14,15,16)(22,23,24)$ \\
& $\sigma_{3}=(1,6)(2,19)(3,10)(4,13)(5,20)(7,8)(9,14)(11,18)(12,21)(15,16)(17,22)(23,24)$
\end{tabular}

As a conclusion we note that Theorem 4.1 and Proposition 4.2 are very far from providing a complete analysis of realizability and exceptionality of candidate covers with associated orbifold candidate of hyperbolic type, because most often the orbifolds involved are not triangular. For instance the candidate surface branched cover $S_{\substack{(4,4,2),(4,-, 2,2),(6,1,1,1,1) \\(10: 1 \rightarrow-\rightarrow-\rightarrow}} S$ considered in the first column of Table 2 in [28] is exceptional, and the associated orbifold candidate $S(6,6,6,6,2,2,2,2) \rightarrow S(4,4,6)$ is hyperbolic but not triangular.

\section{ACKNOWLEDGMENTS}

Part of this work was carried out while the second author was visiting the Université Paul Sabatier in Toulouse and Columbia University in New York. He is grateful to these institutions for support, and to Michel Boileau and Dylan Thurston for 
their warm hospitality and inspiring mathematical discussions. Both authors thank the referee for precious suggestions.

\section{REFERENCES}

[1] K. BARÁNSKI, On realizability of branched coverings on the sphere, Topology Appl. 116 (2001), 279-291. MR1857667 (2002i:57001)

[2] P. Corvaja - C. Petronio - U. Zannier, On certain permutation groups and sums of two squares, arXiv:0810.0591.

[3] A. L. Edmonds - R. S. Kulkarni - R. E. Stong, Realizability of branched coverings of surfaces, Trans. Amer. Math. Soc. 282 (1984), 773-790. MR732119 (85k:57005)

[4] C. L. EzELL, Branch point structure of covering maps onto nonorientable surfaces, Trans. Amer. Math. Soc. 243 (1978), 122-133. MR0500900 (58:18403)

[5] O. Endler, Compact Riemann surfaces with prescribed ramifications and Puiseaux series, Bol. Soc. Brasil. Mat. 2 (1971), 61-64. MR0316702 (47:5249)

[6] G. Francis, Assembling Riemann surfaces with prescribed boundary curves and branch points, Illinois J. Math. 20 (1976), 198-217. MR0402776 (53:6590)

[7] S. M. Gersten, On branched coverings of the 2-sphere by the 2-sphere, Proc. Amer. Math. Soc. 101 (1987), 761-766. MR 911047 (88k:57004)

[8] A. Grothendieck, Esquisse d'un programme (1984). In: "Geometric Galois Actions" (L. Schneps, P. Lochak eds.), 1: "Around Grothendieck's Esquisse d'un Programme", London Math. Soc. Lecture Note Series, Cambridge Univ. Press, Vol. 242, (1997), 5-48. MR.1483107 (99c:14034)

[9] R. M. Guralnick - P. Müller - J. Saxl, "The Rational Function Analogue of a Question of Schur and Exceptionality of Permutation Representations," Mem. Amer. Math. Soc., Vol. 773, Providence, RI (2003), 79 pp. MR.1955160 (2004d:12005)

[10] A. HuRwitz, Über Riemann'sche Flächen mit gegebenen Verzweigungspunkten, Math. Ann. 39 (1891), 1-61. MR 1510692

[11] D. H. Husemoller, Ramified coverings of Riemann surfaces, Duke Math. J. 29 (1962), 167-174. MR0136726 (25:188)

[12] A. G. Khovanski - S. ZDRAVSKovska, Branched covers of $S^{2}$ and braid groups, J. Knot Theory Ramifications 5 (1996), 55-75. MR1373810(97a:57002)

[13] A. D. Mednykh, Nonequivalent coverings of Riemann surfaces with a given ramification type, Sib. Math. Zh. 25 (1984), 120-142. MR754748 (86c:30088)

[14] A. D. Mednykh, Branched coverings of Riemann surfaces whose branch orders coincide with the multiplicity, Commun. Algebra 18 (1990), 1517-1533. MR.1059745 (91e:14024)

[15] S. Monni - J. S. Song - Y. S. Song, The Hurwitz enumeration problem of branched covers and Hodge integrals, J. Geom. Phys. 50 (2004), 223-256. MR2078227(2005h:14130)

[16] A. Okounkov - R. Pandharipande, Gromov-Witten theory, Hurwitz theory, and completed cycles, Ann. of Math. (2) 163 (2006), 517-560. MR.2199225 (2007b:14123)

[17] F. PAKovich, On ramification of Laurent polynomials, to appear in J. Knot Theory Ramifications.

[18] E. Pervova - C. Petronio, On the existence of branched coverings between surfaces with prescribed branch data, Algebr. Geom. Topol. 6 (2006), 1957-1985 (electronic). MR2263056

[19] E. Pervova - C. Petronio, On the existence of branched coverings between surfaces with prescribed branch data, II, J. Knot Theory Ramifications 17 (2008), 787-816. MR2436584

[20] http://www.dm.unipi.it/pages/petronio/public_html/geom_hurw.html

[21] D. Singerman, Subgroups of Fuchsian groups and finite permutation groups, Bull. London. Math. Soc. 2 (1970), 319-323. MR0281805 (43:7519)

[22] R. Тном, L'equivalence d'une fonction différentiable et d'un polynôme, Topology 3 suppl. 2 (1965), 297-307. MR0187249 (32:4702)

[23] W. P. Thurston, The geometry and topology of 3-manifolds, mimeographed notes, Princeton, 1979

[24] J. Wolfart, ABC for polynomials, dessins d'enfants, and uniformization - a survey, in "Elementare und Analytische Zahlentheorie, Proceedings ELAZ-Conference May 24-28, 2004", ed: W. Schwarz, J. Steuding, Steiner Verlag, Stuttgart (2006), 313-345. MR.2310190

[25] U. Zannier, Some remarks on the S-unit equation in function fields, Acta Arith. 64 (1993), 87-98. MR.1220487 (94c:11111) 
[26] U. ZANniER, On Davenport's bound for the degree of $f^{3}-g^{2}$ and Riemann's Existence Theorem, Acta Arith. 71 (1995), 107-137. MR.1339121 (96k:11029a)

[27] U. ZANNIER, Proof of the existence of certain triples of polynomials (after a question of L. Vaserstein and E. Wheland), Rend. Sem. Mat. Univ. Padova 117 (2007). MR2351792

[28] H. Zheng, Realizability of branched coverings of $S^{2}$, Topol. Appl. 153 (2006), 2123-2134. MR2239076 (2007d:57004)

Dipartimento di Matematica, Sapienza Università di Roma, P.le Aldo Moro, 2, 00185 Roma, ITALY

E-mail address: pascali@mat.uniroma1.it

Dipartimento di Matematica Applicata, Università di Pisa, Via Filippo Buonarroti, 1C, 56127 Pisa, Italy

E-mail address: petronio@dm.unipi.it 